\title{
Quantifying people living with HIV who would benefit from an alternative to daily oral therapy: Perspectives from HIV physicians and people living with HIV
}

\author{
Laura Clark ${ }^{1}$, Chitra Karki ${ }^{1}$, Josh Noone ${ }^{1}$, Jenny Scherzer ${ }^{2}$, Martina Bode ${ }^{2}$, Paolo Rizzini ${ }^{3}$, Fabio Vecchio ${ }^{3}$, Laetitia Roustand ${ }^{4}$, \\ Gaelle Nachbaur ${ }^{4}$, Laurent Finkielsztejn ${ }^{5}$, Vasiliki Chounta ${ }^{6}$, Nicolas Van de Velde ${ }^{6}$
}

\section{AFFILIATION}

1 Ipsos Insights LLC, New York, United States

2 ViiV Healthcare Limited, Munich, Germany

3 ViiV Healthcare Limited, Verona, Italy

4 GlaxoSmithKline Pharmaceuticals, Saint Amant les Eaux, France

5 ViiV Healthcare Limited, Rueil-Malmaison, France

6 ViiV Healthcare Limited, Brentford, United Kingdom

CORRESPONDENCE TO

Nicolas Van de Velde. ViiV Healthcare Limited, 980 Great West Road, Brentford, Middlesex, TW8 9GS, United Kingdom.

Popul. Med. 2020;2(October):33
E-mail: nicolas.x.van-de-velde@gsk.com

\section{KEYWORDS}

HIV, adherence, injectable antiretroviral therapy, long-acting HIV treatment, cabotegravir $\mathrm{CAB}$, rilpivirine $\mathrm{RPV}$

Received: 8 August 2020, Accepted: 21 August 2020

https://doi.org/10.18332/popmed/126632

\begin{abstract}
INTRODUCTION Current antiretroviral therapies (ARTs) require daily oral dosing, which is a challenge for some people living with HIV (PLHIV). Measures of treatment needs that are associated with daily oral ARTs have been identified in studies/ interviews with healthcare professionals (HCPs) and PLHIV, and are grouped in four main categories: 1) medical conditions interfering with daily oral administration, 2) suboptimal adherence, 3) confidentiality concerns, and 4) emotional wellbeing related to daily tablet requirements. We quantified these categories to assess the potential benefits of alternatives to daily oral ARTs such as long-acting injectable regimens.

METHODS Two separate online studies were completed by HCPs $(n=120)$ and PLHIV ( $n=698)$ in France, Germany, Italy and the UK, in 2019. HCPs reported the number and percentage of their patients with challenges; unit of analysis among PLHIV was the respondents (\%). Descriptive analyses were performed with R 3.6.1.

RESULTS HIV physicians reported managing a mean of 299
\end{abstract}

( $S D=177$ ) patients, of whom $85.7 \%$ were on ART. Among PLHIV, 98.6\% (688/698) were currently on ART, with mean age of $40.9(S D=12.0)$ years, and $66.4 \%$ men. HCPs estimated that $10-15 \%$ of their patients were affected by each medical condition identified as interfering with daily oral administration. HCPs further estimated that $33.6 \%$ of their patients were suboptimally adherent. 'Non-adherence for any non-medical reason' was reported by HCPs as the primary cause of virologic failure. Of surveyed PLHIV on ART, 43.3\% (298/688) reported hiding their medication and 29.7\% (204/688) indicated they had never shared their HIV status with others. Furthermore, some PLHIV reported that having to remember to dose at the right time every day was stressful $(27.3 \%$; $188 / 688)$ and many saw their tablets as a daily reminder of HIV (45.1\%; 310/688).

CONCLUSIONS A significant proportion of PLHIV struggle with daily oral ART because of medical and/or HIV-specific issues. Alternatives to daily oral ARTs have the potential to improve treatment adherence and quality of life in PLHIV.

\section{INTRODUCTION}

Early initiation of and adherence to antiretroviral therapy (ART) can improve health outcomes among people living with HIV (PLHIV) and prevent onward HIV transmission ${ }^{1-4}$. The global 90-90-90 targets set out by the Joint United Nations Programme on HIV/AIDS (UNAIDS) are to diagnose $90 \%$ of all PLHIV, provide ART for $90 \%$ of those diagnosed, and achieve viral suppression for $90 \%$ of those on ART 5 . Ensuring ART is taken as prescribed is vital to achieving the third target; yet many PLHIV face numerous treatment challenges that can sabotage adherence ${ }^{6-11}$.

Because daily oral ART is currently the sole option for HIV treatment, many problems arise because of either the frequency (i.e. daily) or route of administration (i.e. 
oral) ${ }^{12}$. Daily intake can be a source of concern regarding unintentional disclosure of HIV status or serve as an unwanted reminder of HIV ${ }^{13,14}$. Drug-drug and drug-food interactions, as well as pill aversion, can also complicate daily oral ART ${ }^{12,15-17}$.

With injections every two months, long-acting cabotegravir and rilpivirine (CAB LA + RPV LA) is an innovative HIV treatment regimen designed to provide PLHIV with an alternative that is as effective as daily oral $\mathrm{ART}^{18,19}$. In a 25-country survey of PLHIV, the long-acting attribute was highly favored as a key 'improvement to HIV medicines', second only to improvements in the long-term safety profile of medicines ${ }^{20}$. In that same study, $54.7 \%$ of participants indicated preference for long-acting regimens, especially those with confidentiality concerns, suboptimal adherence, and gastrointestinal ART side effects ${ }^{20}$. In qualitative interviews of patients being treated with CAB LA + RPV LA in the phase IIb clinical trial LATTE-2, patients described the regimen as being more 'discreet' than pills, with less opportunity for stigma, discrimination or undesired disclosure ${ }^{21}$.

Given the anticipated change in treatment paradigm because of the advent of long-acting regimens ${ }^{18,19}$, it is important to quantify problems related to daily oral dosing to determine who may benefit most from long-acting regimens. While some problems have been previously identified ${ }^{12-17,20,21}$, the populations in some of those studies were either PLHIV alone $^{22-24}$ or healthcare providers (HCPs) alone ${ }^{25,26}$. This may not provide a complete picture of problems associated with treatment as attitudes and perceived priorities of patients versus providers may not always converge perfectly. Patients may not fully communicate with their HCPs treatment concerns, including emotional or personal issues, which may be contributing to poor adherence ${ }^{27}$; HCP communication may also be limited, despite its key role ${ }^{28,29}$.

As a prelude to this current study and building upon the work in the literature, we undertook formative research among HIV patients and providers to review, refine and define the categories of problems in relation to daily oral ART dosing, with the aim of better capturing and quantifying them in the present study. From the advisory boards, qualitative interviews, and pilot surveys conducted, four main categories of problems emerged: 1) medical conditions interfering with daily oral administration, 2) suboptimal adherence, 3) confidentiality concerns, and 4) emotional wellbeing related to daily tablet requirements. We explored these objectives from two complementary perspectives - that of PLHIV and HIV physicians - as some categories of unmet needs are better informed by patients (confidentiality, emotional wellbeing) whereas HCPs are better placed to report proportion of patients with specific medical conditions.

\section{METHODS}

\section{Study population}

This study was sponsored by ViiV Healthcare and fielded by Ipsos Healthcare. Web-based surveys of 120 physicians and 698 PLHIV were conducted from June to August 2019 in Germany, Italy, UK, and France. These countries were selected because of their high HIV burden in the European Economic Area $^{30}$, together accounting for over half of all new HIV diagnoses during $2016^{11}$. The logistical challenges of recruiting a statistically robust sample size in smaller European countries made it challenging to perform this study in additional countries. Because of the non-probabilitybased sampling approach used in this study, the target population and the analyzed population are synonymous. Yet, inferences may be transferrable to PLHIV populations in other geographical locations that have similar challenges and shared experiences.

\section{Inclusion criteria}

PLHIV inclusion criteria were: 1) able to confirm selfreported HIV status with either a photo of their HIV medication or their prescription; 2) resident of surveyed country and able to read, speak, and understand the official language; and 3) willing and able to provide electronic informed consent.

HCP inclusion criteria were: 1) board certified or eligible physician directly involved in the treatment of adult PLHIV; 2) practiced internal medicine, HIV medicine, or infectious disease specialty for $\geq 5$ years; 3 ) personally managed $\geq 50$ HIV patients; 4 ) consulted $\geq 15$ HIV patients per week; 5) resident of surveyed country and able to read, speak, and understand the official language; and 6) willing and able to provide electronic informed consent.

\section{Recruitment}

Approximately $60-70 \%$ of all PLHIV in this study came from existing panels; these were pre-screened individuals with a confirmed diagnosis of HIV who were ready and willing to participate in research studies. Use of these existing panels allowed for efficient recruitment of participants in the different countries, based on inclusion criteria. The remaining $30-40 \%$ of participants were actively recruited using different sources affiliated with PLHIV, including nongovernmental organizations, patient support groups; other national, regional, and local charities/support groups; online support groups/communities, social media (Facebook), and direct patient referral (snowball sampling). Among all active recruits, HIV diagnosis was ascertained and confirmed using a photo of PLHIV'S ART medication or prescription with their name on it. All personal identifiable information was discarded after confirming HIV status. To recruit PLHIV, Ipsos Healthcare partnered with two companies, Liberating Research, and Opinion Health, which specialize in recruiting, sourcing, and operating on-going panels/ communities of people who live with different conditions. Liberating Research recruited participants from all four countries, contributing $84.8 \%$ of all study participants combined (66.7\% in France, $80.2 \%$ in Germany, $82.2 \%$ in Italy, and 
$100.0 \%$ in the UK). The remainder came from Opinion Health. Ipsos Healthcare monitored PLHIV recruitment on a weekly basis to ensure that the recruited sample's composition aligned with the national HIV population on key characteristics (age, gender, sexual orientation, and country of origin) ${ }^{11}$.

HCPs were recruited as a purposive sample, a form of non-probability sampling. Panels of previously profiled HCPs with larger caseloads of PLHIV were used. The rationale for oversampling HCPs with many HIV patients was to ensure that we could generate robust estimates for certain outcomes among their managed patients that were assumed to be rare outcomes (e.g. certain medical conditions interfering with oral administration). Secondarily, oversampling HCPs with many HIV patients ensured that providers' perspectives being captured were representative of a diverse array of patients. The participating HCP panelists were sourced from three separate companies that operate on-going panels of providers - Sema, Ipsos (UK/Italy), and Medefield. Sema contributed 8 of 30 HCP participants from France, and 1 of 30 participants from the UK. Ipsos contributed 22 of 30 participants from France, all 30 participants from Italy, and 29 of 30 participants from the UK. Medefield contributed all 30 participants from Germany.

\section{Questionnaire development}

The survey themes were guided by formative research and shaped with input from an advisory panel comprising PLHIV, HIV physicians, and patient advocates. The draft questionnaires developed with the help of this advisory panel, were reviewed by local teams in the respective countries to ensure they were culturally and contextually appropriate (e.g. therapies listed in the questionnaire were consistent with those locally available), and translated into the official language (from English to French, German, and Italian) by medically trained translators. Additional trained translators proofread the original translations for accuracy. The questionnaires were then piloted in each of the four countries amongst a small sample of PLHIV and HCPs separately, to ensure comprehension, clarity, and online usability. During this process, respondents self-completed the online questionnaire while sharing their device screen with (and speaking to) a moderator via WebEx. The online questionnaire was optimized for different types of electronic devices (e.g. mobile, tablet and laptop/desktop) and different operating systems (e.g. IOS, Android).

\section{Survey launch}

Selected participants were sent an electronic mail invitation to participate. Responses were anonymous and could not be linked back to the participant. All patients were required to electronically provide informed consent for participation in this study and meet the inclusion criteria before proceeding to the screener and main survey. At the time of consenting, each participant was assigned a unique identification number (ID). Only the unique ID was recorded and linked to collected data. Upon completion of the questionnaire, respondents were remunerated for their participation (approximately £20 GBP). The weighted average overall response rate was $64.3 \%$ for PLHIV. This study was deemed exempt-research by the Pearl Institutional Review Board (Study number 19-IPSO-125).

\section{Measures}

The survey collected information on several clinical and demographic variables important to interpreting and placing into context the rest of the survey results. Viral suppression was assessed with the question: 'How does your doctor describe your current viral load?'. Those answering 'Undetectable/virologically-suppressed' (i.e. viral load under 50 copies per $\mathrm{mL}$ ) were classified as 'virally suppressed'.

Current ART regimen was based on the core agent reported (non-mutually exclusive categories), as an integrase strand transfer inhibitor (INSTI), a boosted protease inhibitor (PI), or a non-nucleoside reverse transcriptase inhibitor (NNRTI). A past history of resistance to ART was said to be present if this was reported as the reason for the respondent having stopped ART, switched ART, or failed to achieve viral suppression; or if the respondent was currently on Fuzeon (enfuvirtide), whose main indication is for treatment-experienced patients with evidence of HIV-1 replication despite ongoing ART ${ }^{31}$. Detailed measurements of the four broad categories of problems related to daily oral dosing are presented below and in Supplemental Table 1.

\section{Medical conditions interfering with daily oral administration}

In the HCP survey, the following four groups of medical conditions were measured: a) malabsorption; b) gastrointestinal issues interfering with oral administration; c) difficulty in swallowing (e.g. phobia or pill aversion, esophagitis, mechanical obstruction, excluding central nervous system disorders); and d) neurocognitive conditions. For each of these conditions, HCPs quantified the estimated prevalence among HIV patients: 'Based on your experience and knowledge overall'. Individual conditions within the PLHIV survey were grouped into those same four broad categories as the HCP survey (Supplemental Table 1). PLHIV were also asked if they experienced problems while taking ART with other medications, food, or recreational drugs.

\section{Daily oral ART and challenges with adherence}

Adherence was considered 'not only in terms of missed doses but also taking the pills at the right time and under the right conditions without overdosing'. In the HCP survey, physicians estimated what percentage of their patients had suboptimal adherence. In the PLHIV survey, self-reported frequency of failing to take HIV medication exactly as prescribed, as 'Sometimes', 'Often', or 'Very often', was classified as suboptimal adherence. 
Table 1. Healthcare providers (HCPs) who participated in the study - demographic and practice characteristics overall and by country

\begin{tabular}{|c|c|c|c|c|c|}
\hline Characteristics & $\begin{array}{c}\text { Total } \\
(n=120)\end{array}$ & $\begin{array}{l}\text { France } \\
(n=30)\end{array}$ & $\begin{array}{l}\text { Germany } \\
(\mathrm{n}=\mathbf{3 0})\end{array}$ & $\begin{array}{c}\text { Italy } \\
(\mathrm{n}=30)\end{array}$ & $\begin{array}{c}\text { UK } \\
(n=30)\end{array}$ \\
\hline \multicolumn{6}{|l|}{$\begin{array}{l}\text { Characteristics of physicians (i.e. individual } \\
\text { HCP respondents as unit of analyses) }\end{array}$} \\
\hline \multicolumn{6}{|l|}{ Gender } \\
\hline Women & 31.7 & 36.7 & 23.3 & 36.7 & 30.0 \\
\hline Men & 65.8 & 56.7 & 73.3 & 63.3 & 70.0 \\
\hline Prefer not to say & 2.5 & 6.7 & 3.3 & 0.0 & 0.0 \\
\hline \multicolumn{6}{|l|}{ Primary medical specialty } \\
\hline Internal medicine & 5.0 & 13.3 & 6.7 & 0.0 & 0.0 \\
\hline HIV/AIDS & 36.7 & 50.0 & 36.7 & 6.7 & 53.3 \\
\hline Infectious diseases & 55.8 & 36.7 & 56.7 & 93.3 & 36.7 \\
\hline Genito-urinary medicine & 2.5 & 0.0 & 0.0 & 0.0 & 10.0 \\
\hline \multicolumn{6}{|l|}{ Primary practice setting } \\
\hline Hospital & 1.7 & 0.0 & 6.7 & 0.0 & 0.0 \\
\hline University or medical school-based hospital & 14.2 & 0.0 & 56.7 & 0.0 & 0.0 \\
\hline District general hospital & 14.2 & 0.0 & 0.0 & 0.0 & 56.7 \\
\hline General hospital & 36.7 & 73.3 & 0.0 & 73.3 & 0.0 \\
\hline Private practice - HIV specialist & 9.2 & 0.0 & 36.7 & 0.0 & 0.0 \\
\hline Outpatient clinic & 0.8 & 0.0 & 0.0 & 3.3 & 0.0 \\
\hline Genito-urinary medicine (GUM) clinic & 5.0 & 0.0 & 0.0 & 0.0 & 20.0 \\
\hline Other & 18.3 & 26.7 & 0.0 & 23.3 & 23.3 \\
\hline \multicolumn{6}{|l|}{ Primary practice location } \\
\hline Major metropolitan area & 58.3 & 63.3 & 66.7 & 66.7 & 36.7 \\
\hline Urban area & 32.5 & 23.3 & 33.3 & 30.0 & 43.3 \\
\hline Suburb of a large city & 7.5 & 10.0 & 0.0 & 0.0 & 20.0 \\
\hline Small city & 1.7 & 3.3 & 0.0 & 3.3 & 0.0 \\
\hline \multicolumn{6}{|l|}{ Current staff grade } \\
\hline Professor & 8.3 & 16.7 & 6.7 & 3.3 & 6.7 \\
\hline Consultant & 45.0 & 60.0 & 30.0 & 3.3 & 86.7 \\
\hline Associate Specialist & 22.5 & 20.0 & 63.3 & 3.3 & 3.3 \\
\hline Specialist Registrar & 24.2 & 3.3 & 0.0 & 90.0 & 3.3 \\
\hline \multicolumn{6}{|l|}{ Years of experience } \\
\hline Mean (SD) & $20.0(9.7)$ & $25.0(12.8)$ & $15.7(8.9)$ & $20.6(6.3)$ & $18.6(7.2)$ \\
\hline \multicolumn{6}{|l|}{ Years in current practice } \\
\hline Mean (SD) & $17.8(6.0)$ & $20.1(6.3)$ & $14.6(5.3)$ & $19.3(5.4)$ & $17.33(5.7)$ \\
\hline \multicolumn{6}{|c|}{$\begin{array}{l}\text { Characteristics of patients managed by HCPs } \\
\text { (i.e. discreet patient-related responses as unit } \\
\text { of analyses) }\end{array}$} \\
\hline \multicolumn{6}{|l|}{$\begin{array}{l}\text { Number of HIV patients currently under } \\
\text { management }\end{array}$} \\
\hline Mean (SD) & $299.1(176.8)$ & $276.5(134.8)$ & $181.0(199.2)$ & $377.8(192.2)$ & $361.2(183.2)$ \\
\hline \multicolumn{6}{|l|}{ HIV patients from the country of report } \\
\hline Mean (SD) & $58.6(29.7)$ & $46.1(26.1)$ & $45.9(31.2)$ & $78.8(11.5)$ & $63.87(32.7)$ \\
\hline
\end{tabular}


Table 1. Continued

\begin{tabular}{|c|c|c|c|c|c|}
\hline Characteristics & $\begin{array}{c}\text { Total } \\
(n=120)\end{array}$ & $\begin{array}{l}\text { France } \\
(n=30)\end{array}$ & $\begin{array}{l}\text { Germany } \\
(n=30)\end{array}$ & $\begin{array}{c}\text { Italy } \\
(n=30)\end{array}$ & $\begin{array}{c}\text { UK } \\
(n=30)\end{array}$ \\
\hline \multicolumn{6}{|c|}{$\begin{array}{l}\text { HIV patients who are homosexual/gay/ } \\
\text { lesbian }\end{array}$} \\
\hline Mean (SD) & $51.7(24.6)$ & 37.9 (14.3) & $64.3(19.8)$ & $65.1(25.5)$ & 39.7 (23.2) \\
\hline \multicolumn{6}{|c|}{ HIV patients currently on ART } \\
\hline Mean (SD) & 85.7 (15.9) & $89.6(10.7)$ & $85.7(14.7)$ & $87.8(8.8)$ & $79.60(23.8)$ \\
\hline \multicolumn{6}{|c|}{$\begin{array}{l}\text { HIV patients not currently on ART and } \\
\text { treatment naïve }\end{array}$} \\
\hline Mean (SD) & $9.4(10.7)$ & $7.7(8.3)$ & $8.2(9.5)$ & $8.4(6.8)$ & $13.2(15.5)$ \\
\hline \multicolumn{6}{|c|}{$\begin{array}{l}\text { HIV patients not currently on ART and } \\
\text { treatment experienced }\end{array}$} \\
\hline Mean (SD) & $5.0(6.7)$ & $2.7(3.7)$ & $6.1(6.6)$ & $3.8(2.9)$ & $7.2(10.1)$ \\
\hline \multicolumn{6}{|c|}{$\begin{array}{l}\text { HIV patients currently on ART and virally } \\
\text { suppressed }\end{array}$} \\
\hline Mean (SD) & 83.9 (19.3) & $88.8(8.7)$ & $81.0(23.4)$ & $87.70(10.4)$ & $78.2(26.9)$ \\
\hline \multicolumn{6}{|c|}{$\begin{array}{l}\text { HIV patients currently on ART, virally } \\
\text { suppressed and no history of treatment } \\
\text { failure }\end{array}$} \\
\hline Mean (SD) & $66.4(22.1)$ & $69.0(17.2)$ & $73.6(25.6)$ & $59.0(19.3)$ & $63.9(23.6)$ \\
\hline
\end{tabular}

ART: antiretroviral therapy. HCP: healthcare provider. SD: standard deviation. Numbers are column \% for each characteristic, unless otherwise stated.

Confidentiality concerns associated with daily oral ART Similar questions were asked in the HCP and PLHIV surveys regarding patients' attitudes and behaviors towards sharing of their HIV status with others, hiding of HIV medication to prevent unwanted disclosure, and perceived stigma. HCPs who reported the perceived frequency of privacy concerns among HIV patients as 'Often' were classified as perceiving it as prevalent. Among PLHIV, responses of either 'Yes', or reported frequency of 'Sometimes'/'Often'/'Very Often' were classified as positive responses.

Emotional wellbeing related to daily tablet requirements The HCP and PLHIV questionnaires assessed treatmentrelated challenges, including patients' emotional wellbeing as well as their concerns about HIV and/or HIV treatment. Covered issues included dosing schedule, perceived short-, intermediate-, and long-term impacts of treatment, concerns about transmitting HIV, and sexual wellbeing.

\section{Statistical analyses}

Because these four countries are well-defined geographically and have similar HIV profiles, we conducted pooled analyses of the data ${ }^{30}$. The surveys were all standardized and fielded at the same time, minimizing measurement and time biases. Statistical comparisons of key characteristics showed no significant differences across countries on several key characteristics including age, marital status, and year of diagnosis. Pooled analyses allowed for increased precision of subgroup estimates.

For the HCP survey, physicians provided their best estimate for the proportion of HIV patients that met a characteristic of interest. For the PLHIV survey, the individual respondent was the unit of analysis, and unless stated otherwise, all analyses were among those currently on ART ( $\mathrm{n}=688)$. Within-group statistical comparisons were performed using $\chi^{2}$ at $\mathrm{p}<0.05$. All statistical analyses were conducted using R Version 3.6.3.

\section{RESULTS}

Half of the participating HCPs worked in general hospitals (including district general hospitals, 50.9\%), 14.2\% worked in university or medical school-based hospitals, and $9.2 \%$ were in private practice. The remainder (39.9\%) worked in outpatient clinics, genito-urinary medicine clinics, or other settings. HCPs reported managing a mean of 299 (SD=177) HIV patients, of whom $85.7 \%$ were reportedly on ART. Other HCP characteristics and those of their managed patients are given in Table 1.

Of all PLHIV respondents, 98.6\% (688/698) were currently on ART. Mean age and duration of HIV among PLHIV on treatment were $40.9(\mathrm{SD}=12.0)$ and $11.8(\mathrm{SD}=9.6)$ years, respectively. Most PLHIV on treatment were: homosexual $(60.6 \%)$; men $(66.4 \%)$; with a partner $(52.5 \%)$; college educated (58.6\%); employed (68.3\%); living in metropolitan areas (69.5\%); virally suppressed (89.4\%); diagnosed prior to 2017 (87.2\%); and native born (62.4\%) (Table 2). 
Medical conditions interfering with daily oral administration HCP-reported prevalence for different medical conditions among HIV patients was: malabsorption (9.8\%); gastrointestinal conditions interfering with oral administration (10.4\%); difficulty swallowing (9.7\%); and neurocognitive conditions (11.6\%) (Figure 1). Categorical distributions of HCP perceived burden of various conditions, depicted in Figure 2, revealed that none to only a small fraction of HCPs perceived any of the assessed problems as being nonexistent (i.e. estimated prevalence of 0\%) among HIV patients. Except for malabsorption, PLHIV reported higher rates of these conditions than the corresponding
HCP-reported estimates (Figure 1). Prevalence of specific neurocognitive/mental conditions by PLHIV report (nonmutually exclusive) included depression (24.3\%; 167/688), anxiety $(20.9 \% ; 144 / 688)$, and dementia $(0.6 \% ; 4 / 688)$ (Figure 3). Overall, 18.6\% (128/688) of PLHIV reported being diagnosed with a gastrointestinal condition interfering with oral ART administration. Furthermore, $42.5 \%$ of PLHIV reported that 'I must take food at the same time as my HIV treatment', 23.9\% indicated that 'I cannot take antacids, PPIs or $\mathrm{H} 2$ blockers to relieve stomach issues along with my HIV treatment', and $16.1 \%$, reported 'I cannot take another drug at the same time as my HIV treatment' (Table 3). In

Table 2. Characteristics of adults living with HIV on antiretroviral therapy in four European countries as well as the percentage reporting various medical conditions interfering with daily oral ART dosing, 2019

\begin{tabular}{|c|c|c|c|c|c|c|c|c|c|}
\hline \multirow[t]{2}{*}{ Characteristics } & \multirow{2}{*}{\begin{tabular}{c|} 
Distribution \\
n (\%)
\end{tabular}} & \multicolumn{2}{|c|}{ Malabsorption } & \multicolumn{2}{|c|}{$\begin{array}{l}\text { Gastrointestinal } \\
\text { conditions } \\
\text { interfering with } \\
\text { oral ART }\end{array}$} & \multicolumn{2}{|c|}{$\begin{array}{l}\text { Dysphagia } \\
\text { (difficulty } \\
\text { swallowing) }\end{array}$} & \multicolumn{2}{|c|}{$\begin{array}{l}\text { Neurocognitive } \\
\text { conditions }\end{array}$} \\
\hline & & $\%$ & $\mathbf{p}$ & $\%$ & $\mathbf{p}$ & $\%$ & $\mathbf{p}$ & $\%$ & $\mathbf{p}$ \\
\hline Total & $688(100.0)$ & 8.1 & & 18.6 & & 17.9 & & 35.5 & \\
\hline \multicolumn{10}{|l|}{ Gender } \\
\hline Women & $229(33.3)$ & 8.3 & \multirow{3}{*}{$\begin{array}{c}\chi^{2}(2)=0.19 \\
p=0.911\end{array}$} & 21.8 & \multirow{3}{*}{$\begin{array}{c}\chi^{2}(2)=2.75 \\
p=0.253\end{array}$} & 32.3 & \multirow{3}{*}{$\begin{array}{c}\chi^{2}(2)=48.88 \\
p<0.001\end{array}$} & 33.2 & \multirow{3}{*}{$\begin{array}{c}\chi^{2}(2)=4.31 \\
p=0.116\end{array}$} \\
\hline Men & $457(66.4)$ & 8.1 & & 17.1 & & 10.7 & & 36.3 & \\
\hline Other & $2(0.3)$ & १ & & Т & & Т & & Т & \\
\hline \multicolumn{10}{|c|}{ Sexual orientation } \\
\hline Heterosexual & 233 (33.9) & 6.4 & \multirow{3}{*}{$\begin{array}{c}\chi^{2}(2)=3.91 \\
p=0.141\end{array}$} & 20.6 & \multirow{3}{*}{$\begin{array}{c}\chi^{2}(2)=1.46 \\
p=0.481\end{array}$} & 30.0 & \multirow{3}{*}{$\begin{array}{c}\chi^{2}(2)=41.59 \\
p<0.001\end{array}$} & 32.2 & \multirow{3}{*}{$\begin{array}{c}\chi^{2}(2)=2.70 \\
p=0.260\end{array}$} \\
\hline Homosexual & $417(60.6)$ & 8.4 & & 18.0 & & 10.3 & & 36.5 & \\
\hline Other & $38(5.5)$ & 15.8 & & 13.2 & & 26.3 & & 44.7 & \\
\hline \multicolumn{10}{|l|}{ Age (years) } \\
\hline$<50$ & $484(70.4)$ & 7.6 & \multirow{2}{*}{$\begin{array}{c}\chi^{2}(1)=0.54 \\
p=0.465\end{array}$} & 18.2 & \multirow{2}{*}{$\begin{array}{c}\chi^{2}(1)=0.19 \\
p=0.661\end{array}$} & 21.1 & \multirow{2}{*}{$\begin{array}{c}\chi^{2}(1)=11.36 \\
p=0.001\end{array}$} & 34.7 & \multirow{2}{*}{$\begin{array}{c}\chi^{2}(1)=0.41 \\
p=0.524\end{array}$} \\
\hline$\geq 50$ & 204 (29.7) & 9.3 & & 19.6 & & 10.3 & & 37.3 & \\
\hline \multicolumn{10}{|l|}{ Education level } \\
\hline Postgraduate & $134(20.0)$ & 10.4 & \multirow{4}{*}{$\begin{array}{c}\chi^{2}(3)=2.72 \\
p=0.436\end{array}$} & 26.1 & \multirow{4}{*}{$\begin{array}{c}\chi^{2}(3)=6.86 \\
p=0.076\end{array}$} & 19.4 & \multirow{4}{*}{$\begin{array}{c}\chi^{2}(3)=2.97 \\
p=0.396\end{array}$} & 34.3 & \multirow{4}{*}{$\begin{array}{c}\chi^{2}(3)=3.08 \\
p=0.380\end{array}$} \\
\hline College & 392 (58.6) & 6.6 & & 18.4 & & 19.1 & & 33.4 & \\
\hline Secondary & $99(14.8)$ & 10.1 & & 14.1 & & 12.1 & & 42.4 & \\
\hline Other & $44(6.6)$ & 9.1 & & 13.6 & & 15.9 & & 38.6 & \\
\hline \multicolumn{10}{|l|}{ Employment } \\
\hline Employed & 457 (68.3) & 7.0 & \multirow{2}{*}{$\begin{array}{c}\chi^{2}(1)=2.22 \\
p=0.136\end{array}$} & 17.9 & \multirow{2}{*}{$\begin{array}{c}\chi^{2}(1)=1.02 \\
p=0.314\end{array}$} & 16.8 & $\chi^{2}(1)=1.16$ & 28.7 & $\chi^{2}(1)=27.61$ \\
\hline Non-employed & 212 (31.7) & 10.4 & & 21.2 & & 20.3 & $\mathrm{p}=0.281$ & 49.5 & $\mathrm{p}<0.001$ \\
\hline Country & & & & & & & & & \\
\hline France & 144 (20.9) & 13.9 & $\chi^{2}(3)=8.58$ & 34.7 & $\chi^{2}(3)=50.29$ & 33.3 & $\chi^{2}(3)=42.79$ & 38.9 & $\chi^{2}(3)=15.04$ \\
\hline Germany & 198 (28.8) & 6.1 & $\mathrm{p}=0.035$ & 8.6 & $\mathrm{p}<0.001$ & 6.6 & $\mathrm{p}<0.001$ & 32.8 & $\mathrm{p}=0.002$ \\
\hline Italy & $150(21.8)$ & 8.0 & & 26.0 & & 21.3 & & 24.7 & \\
\hline UK & $196(28.5)$ & 6.1 & & 11.2 & & 15.3 & & 43.9 & \\
\hline ART formulatior & & & & & & & & & \\
\hline Single table & $381(55.4)$ & 7.9 & $\chi^{2}(1)=0.08$ & 17.1 & $\chi^{2}(1)=1.34$ & 15.2 & $\chi^{2}(1)=4.10$ & 30.7 & $\chi^{2}(1)=8.44$ \\
\hline Multi-tablet & 307 (44.6) & 8.5 & $\mathrm{p}=0.777$ & 20.5 & $\mathrm{p}=0.246$ & 21.2 & $\mathrm{p}=0.043$ & 41.4 & $\mathrm{p}=0.004$ \\
\hline
\end{tabular}


Table 2. Continued

\begin{tabular}{|c|c|c|c|c|c|c|c|c|c|}
\hline \multirow[t]{2}{*}{ Characteristics } & \multirow{2}{*}{$\begin{array}{c}\text { Distribution } \\
\text { n (\%) }\end{array}$} & \multicolumn{2}{|c|}{ Malabsorption } & \multicolumn{2}{|c|}{$\begin{array}{l}\text { Gastrointestinal } \\
\text { conditions } \\
\text { interfering with } \\
\text { oral ART }\end{array}$} & \multicolumn{2}{|c|}{$\begin{array}{l}\text { Dysphagia } \\
\text { (difficulty } \\
\text { swallowing) }\end{array}$} & \multicolumn{2}{|c|}{$\begin{array}{l}\text { Neurocognitive } \\
\text { conditions }\end{array}$} \\
\hline & & $\%$ & $\mathbf{p}$ & $\%$ & $\mathbf{p}$ & $\%$ & $\mathbf{p}$ & $\%$ & $\mathbf{p}$ \\
\hline \multicolumn{10}{|l|}{$\begin{array}{l}\text { Self-reported viral } \\
\text { status }\end{array}$} \\
\hline Non-suppressed & $73(10.6)$ & 12.3 & \multirow{2}{*}{$\begin{array}{c}\chi^{2}(1)=1.92 \\
p=0.166\end{array}$} & 45.2 & $\chi^{2}(1)=38.16$ & 60.3 & \multirow{2}{*}{$\begin{array}{c}\chi^{2}(1)=99.98 \\
p<0.001\end{array}$} & 38.4 & \multirow{2}{*}{$\begin{array}{c}\chi^{2}(1)=0.30 \\
p=0.585\end{array}$} \\
\hline Suppressed & $615(89.4)$ & 7.6 & & 15.4 & $\mathrm{p}<0.001$ & 12.8 & & 35.1 & \\
\hline \multicolumn{10}{|l|}{ ART side effects ${ }^{a}$} \\
\hline None reported & $344(50.0)$ & 5.2 & \multirow{3}{*}{$\begin{array}{c}\chi^{2}(2)=10.29 \\
p=0.006\end{array}$} & 11.6 & \multirow{3}{*}{$\begin{array}{c}\chi^{2}(2)=32.75 \\
p<0.001\end{array}$} & 2.0 & \multirow{3}{*}{$\begin{array}{c}\chi^{2}(2)=208.40 \\
p<0.001\end{array}$} & 27.9 & \multirow{3}{*}{$\begin{array}{c}\chi^{2}(2)=17.28 \\
p<0.001\end{array}$} \\
\hline $\begin{array}{l}\text { Non-gastrointestinal } \\
\text { only }\end{array}$ & $96(14.0)$ & 7.3 & & 14.6 & & 2.1 & & 41.7 & \\
\hline Gastrointestinal & $248(36.1)$ & 12.5 & & 29.8 & & 46.0 & & 43.5 & \\
\hline \multicolumn{10}{|l|}{ HIV diagnosis year } \\
\hline 2017-19 & $88(12.8)$ & 1.1 & \multirow{3}{*}{$\begin{array}{c}\chi^{2}(2)=10.07 \\
p=0.006\end{array}$} & 9.1 & \multirow{3}{*}{$\begin{array}{c}\chi^{2}(2)=7.05 \\
p=0.029\end{array}$} & 17.0 & \multirow{3}{*}{$\begin{array}{c}\chi^{2}(2)=8.37 \\
p=0.015\end{array}$} & 17.0 & \multirow{3}{*}{$\begin{array}{c}\chi^{2}(2)=15.46 \\
p<0.001\end{array}$} \\
\hline $2010-16$ & $286(41.6)$ & 7.0 & & 21.7 & & 22.7 & & 36.7 & \\
\hline Pre-2010 & $314(45.6)$ & 11.1 & & 18.5 & & 13.7 & & 39.5 & \\
\hline \multicolumn{10}{|l|}{$\begin{array}{l}\text { NNRTI-containing } \\
\text { ART }^{\text {b }}\end{array}$} \\
\hline No & $450(65.4)$ & 7.1 & \multirow{2}{*}{$\begin{array}{c}\chi^{2}(1)=1.84 \\
p=0.175\end{array}$} & 17.1 & \multirow{2}{*}{$\begin{array}{c}\chi^{2}(1)=1.92 \\
p=0.166\end{array}$} & 14.9 & \multirow{2}{*}{$\begin{array}{c}\chi^{2}(1)=7.92 \\
p=0.005\end{array}$} & 33.6 & \multirow{2}{*}{$\begin{array}{c}\chi^{2}(1)=2.07 \\
p=0.150\end{array}$} \\
\hline Yes & $238(34.6)$ & 10.1 & & 21.4 & & 23.5 & & 39.1 & \\
\hline \multicolumn{10}{|l|}{$\begin{array}{l}\text { Entry inhibitor- } \\
\text { containing ART }\end{array}$} \\
\hline No & $661(96.1)$ & 29.6 & $\chi^{2}(1)=17.36$ & 18.5 & $\chi^{2}(1)=0.24$ & 17.2 & $\chi^{2}(1)=4.57$ & 35.4 & $\chi^{2}(1)=0.03$ \\
\hline Yes & $27(3.9)$ & & $\mathrm{p}<0.001$ & 22.2 & $\mathrm{p}=0.622$ & 33.3 & $\mathrm{p}=0.032$ & 37.0 & $\mathrm{p}=0.862$ \\
\hline \multicolumn{10}{|l|}{$\begin{array}{l}\text { INSTI-containing } \\
\text { ART }^{d}\end{array}$} \\
\hline No & $300(43.6)$ & 7.0 & \multirow{2}{*}{$\begin{array}{c}\chi^{2}(1)=0.92 \\
p=0.336\end{array}$} & 16.0 & \multirow{2}{*}{$\begin{array}{c}\chi^{2}(1)=2.38 \\
p=0.123\end{array}$} & 18.7 & \multirow{2}{*}{$\begin{array}{c}\chi^{2}(1)=0.22 \\
p=0.635\end{array}$} & 34.3 & $\chi^{2}(1)=0.30$ \\
\hline Yes & $388(56.4)$ & 9.0 & & 20.6 & & 17.3 & & 36.3 & $\mathrm{p}=0.585$ \\
\hline $\begin{array}{l}\text { Protease inhibitor- } \\
\text { containing } \mathrm{ART}^{\mathrm{e}}\end{array}$ & & & & & & & & & \\
\hline No & $538(78.2)$ & 7.8 & $\chi^{2}(1)=0.37$ & 17.5 & $\chi^{2}(1)=2.09$ & 14.7 & $\chi^{2}(1)=17.14$ & 35.5 & $\chi^{2}(1)<0.01$ \\
\hline Yes & $150(21.8)$ & 9.3 & $\mathrm{p}=0.545$ & 22.7 & $p=0.148$ & 29.3 & $\mathrm{p}<0.001$ & 35.3 & $\mathrm{p}=0.970$ \\
\hline Past ART resistance $^{\mathrm{f}}$ & & & & & & & & & \\
\hline No & $603(87.7)$ & 6.8 & $\chi^{2}(1)=11.72$ & 15.4 & $\chi^{2}(1)=32.63$ & 15.1 & $\chi^{2}(1)=25.82$ & 33.2 & $\chi^{2}(1)=11.26$ \\
\hline Yes & 85 (12.4) & 17.6 & $\mathrm{p}<0.001$ & 41.2 & $\mathrm{p}<0.001$ & 37.6 & $\mathrm{p}<0.001$ & 51.8 & $\mathrm{p}=0.001$ \\
\hline
\end{tabular}

ART: antiretroviral therapy (the different classes presented in table are not mutually exclusive); NNRTI: non-nucleoside reverse transcriptase inhibitor. INSTI: integrase strand transfer inhibitor. a A history of major ART side effects was said to be present if the respondent reported a past adverse effect from HIV medication (e.g. 'stomach/ gastric problems because of the medication' or 'difficulties taking my HIV treatment as I was having too many side effects'), that led to stopping ART, switching ART, or failing to achieve viral suppression from poor adherence, all because of the side effects. b NNRTI-containing regimens included 'Atripla ${ }^{\circledR}$ or generics (emtricitabine/ efavirenz/tenofovir disoproxil fumarate)'; 'Delstrigo (doravirine/lamivudine/tenofovir disoproxil fumarate)'; 'Edurant (rilpivirine)'; 'Eviplera (emtricitabine/rilpivirine/ tenofovir-disoproxil fumarate)'; 'Viramune or generics (Nevirapin)'; 'Sustiva or generics(efavirenz)'; 'Odefsey (emtricitabine/rilpivirine/tenofovir alafenamide)'; or 'Pifeltro (doravirine)'. c Entry inhibitor-containing regimens included 'Celsentri (maraviroc)'; 'Fuzeon (enfuvirtide)' or 'Fostemsavir'. d INSTI-containing regimens included 'Genvoya (elvitegravir/cobicistat/emtricitabine/tenofovir alafenamide)'; 'Tivicay (dolutegravir)'; 'Triumeq (dolutegravir/abacavir/lamivudine)'; 'Isentress (raltegravir)'; 'Juluca (dolutegravir/rilpivirine)'; 'Stribild (elvitegravir/cobicistat/emtricitabine/tenofovir disoproxil fumarate); or 'Biktarvy (bictegravir/emtricitabine/tenofovir alafenamide)'. e Protease inhibitor-containing regimens included 'Kaletra (lopinavir/ritonavir)'; 'Evotaz (atazanavir/cobicistat)'; 'Prezista (darunavir)'; 'Reyataz (atazanavir)'; 'Rezolsta (darunavir/cobicistat)'; or 'Symtuza (darunavir/emtricitabine/tenofovir alafenamide)'. f A history of resistance to ART was said to be present if this was reported as the reason for the respondent having stopped ART, switched ART, or failed to achieve viral suppression; or if the respondent was currently on Fuzeon (enfuvirtide), whose main indication is for treatment-experienced patients with evidence of HIV-1 replication despite ongoing ART. ๆ Results not presented because of small sample size. 
Figure 1. A comparison of HCP and PLHIV-reported prevalence estimates for various medical, emotional, and psychosocial challenges to daily oral administration of HIV medicines among adults living with HIV on antiretroviral therapy in four European countries, 2019
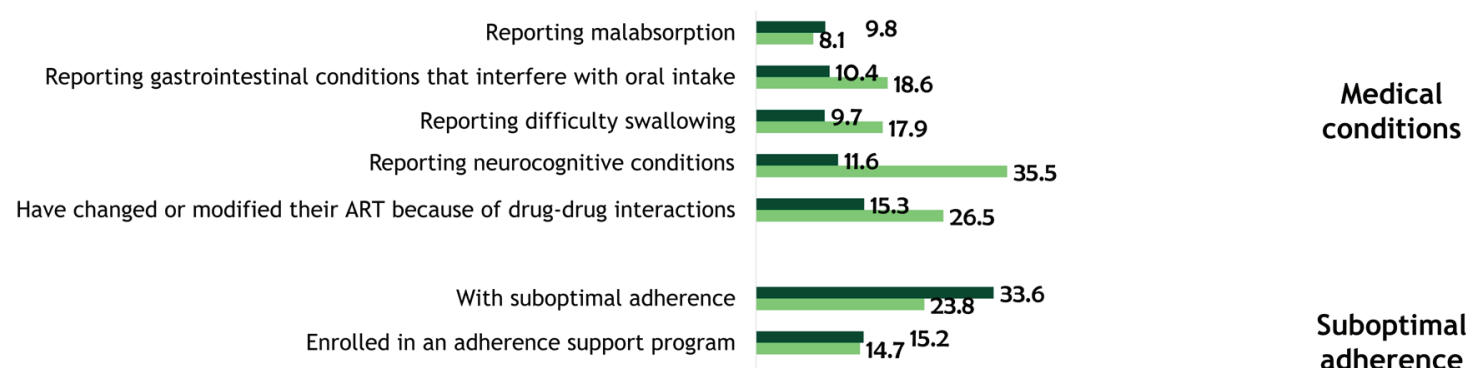

Suboptimal adherence

Ever hid/disguised their HIV pills to avoid sharing their HIV status Reported they have not shared their HIV status with anyone Open in talking about their HIV

Only few people know about their status and won't share even if asked Limited what they told others about their HIV Worried about family members finding out about their HIV treatment Worried about workmates finding out about their HIV treatment Were afraid to tell others about their HIV treatment Worried about losing job if others found they had HIV

Worried about someone seeing their HIV pills at home or on them Worried about friends finding about their HIV while on holiday together Worried about airport security/customs finding their HIV pills while traveling

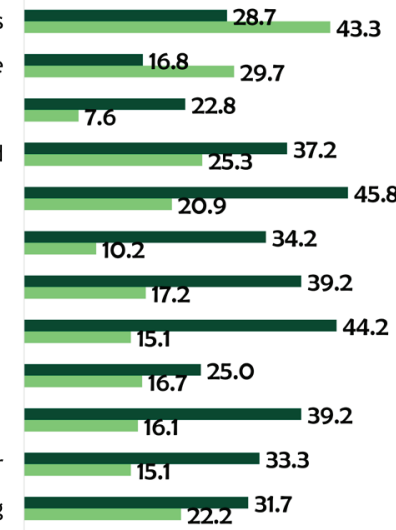

Privacy concerns

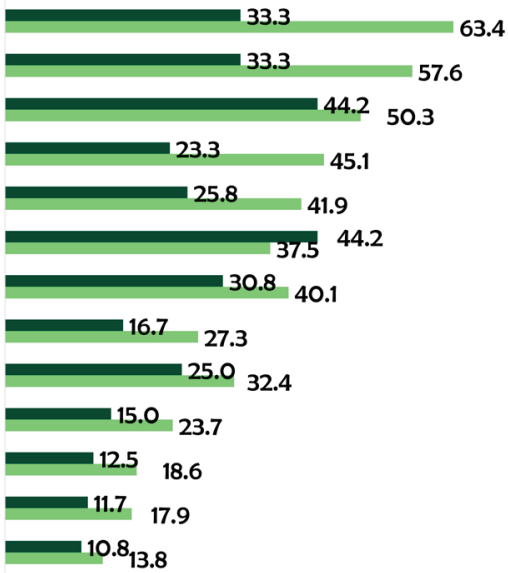

Emotional challenges Indicated that being tied to daily HIV treatment limited their day-to-day life Indicated that their daily oral ART disrupted their day-to-day leisure activities Indicated that their daily dosing schedule was stressful indicated that their daily oral ART disrupted their day-to-day work routine Worried about long-term effects of HIV treatments
Would rather not have to think about taking HIV pills every day
Wished HIV treatment was a smaller part of their life
Daily oral ART reminded them of HIV/bad memories Worried about not being suppressed anymore because of suboptimal adherence Wished to forget about having HIV Worried about missing doses / taking it at the wrong time Having to remember to dose at the right time every day stressful Worried about transmitting HIV and this was impacting their sex life 0 20 $\begin{array}{cc}40 & 60 \\ \text { PERCENTAGE, } \%\end{array}$

- Estimate by HCPs regarding patients with HIV, \% — Self-report by PLHIV, \%

Note: ART: antiretroviral therapy; HCP: healthcare providers; PLHIV: people living with HIV. To align with the HIV physician survey, the percentage who have changed or modified their ART as presented in the figure for people living with HIV was based on affirmative response to either of the following: 'I need additional monitoring when I take other medications on top of my HIV treatment', or 'I had to change at least one drug of my HIV treatment to avoid issues/complications with another drug I had to take at the same time'.

addition, 19.1\% of PLHIV indicated that 'I need additional monitoring when I take other medications on top of my HIV treatment' while $15.4 \%$ ever 'had to change at least one drug of my HIV treatment to avoid issues/complications with another drug' (Table 3). These medical issues were associated with treatment avoidance behavior among PLHIV.
For example, $14.7 \%(101 / 688)$ reported they missed their ART dose because they 'have trouble swallowing pills', $16.3 \%$ $(112 / 688)$ because of 'a problem taking pills at specified times (with meals, on empty stomach etc.)', and $11.2 \%$ (77/688) because they 'were taking another medication'. PLHIV reporting 'I cannot take another drug at the same 
Figure 2. HCP-reported distribution of their patients with different medical conditions and suboptimal adherence, overall and by country, 2019

\begin{tabular}{|c|c|c|c|c|c|c|}
\hline & & Dis & tion of percei & prevalence a & ng HIV physici & s, \% \\
\hline $\begin{array}{l}\text { HIV Physic } \\
\text { prevale } \\
\text { condition amo }\end{array}$ & $\begin{array}{l}\text { Is' estimated } \\
\text { of specified } \\
\text { HIV patients }\end{array}$ & $\begin{array}{l}\text { Malabsorp- } \\
\text { tion }\end{array}$ & $\begin{array}{l}\text { Interferring } \\
\text { gastro- } \\
\text { intestinal } \\
\text { conditions }\end{array}$ & Dysphagia & $\begin{array}{l}\text { Central } \\
\text { nervous } \\
\text { system } \\
\text { conditions }\end{array}$ & $\begin{array}{l}\text { Suboptimal } \\
\text { adherence }\end{array}$ \\
\hline & $0 \%$ & 15.0 & 5.8 & 6.7 & 2.5 & 0.0 \\
\hline & $1-10 \%$ & 59.2 & 64.2 & 65.8 & 65.8 & 35.0 \\
\hline & $11-20 \%$ & 15.8 & 20.8 & 18.3 & 20.0 & 17.5 \\
\hline HIV physicians (N & $21-30 \%$ & 4.2 & 5.0 & 5.8 & 5.0 & 14.2 \\
\hline $\begin{array}{c}\text { HIV physiclans (N } \\
=120)\end{array}$ & $31-40 \%$ & 1.7 & 1.7 & 0.0 & 2.5 & 1.7 \\
\hline & $41-50 \%$ & 0.8 & 0.8 & 0.8 & 2.5 & 5.8 \\
\hline & $51 \%-100 \%$ & 3.3 & 1.7 & 2.5 & 1.7 & 25.8 \\
\hline & $0 \%$ & 20.0 & 6.7 & 6.7 & 3.3 & 0.0 \\
\hline & $1-10 \%$ & 53.3 & 56.7 & 56.7 & 43.3 & 6.7 \\
\hline & $11-20 \%$ & 20.0 & 33.3 & 26.7 & 36.7 & 3.3 \\
\hline $\begin{array}{l}\text { HIV physiclans in } \\
\text { France }(\mathrm{N}=30)\end{array}$ & $21-30 \%$ & 0.0 & 0.0 & 3.3 & 3.3 & 3.3 \\
\hline & $31-40 \%$ & 0.0 & 3.3 & 0.0 & 6.7 & 0.0 \\
\hline & $41-50 \%$ & 0.0 & 0.0 & 0.0 & 3.3 & 3.3 \\
\hline & $51-100 \%$ & 6.7 & 0.0 & 6.7 & 3.3 & 83.3 \\
\hline & $0 \%$ & 23.3 & 3.3 & 10.0 & 3.3 & 0.0 \\
\hline & $1-10 \%$ & 53.3 & 63.3 & 73.3 & 73.3 & 23.3 \\
\hline & $11-20 \%$ & 16.7 & 23.3 & 6.7 & 20.0 & 26.7 \\
\hline $\begin{array}{l}\text { HIV physicians in } \\
\text { Germany }(\mathrm{N}=30)\end{array}$ & $21-30 \%$ & 0.0 & 3.3 & 6.7 & 3.3 & 26.7 \\
\hline & $31-40 \%$ & 0.0 & 0.0 & 0.0 & 0.0 & 3.3 \\
\hline & $41-50 \%$ & 0.0 & 0.0 & 0.0 & 0.0 & 6.7 \\
\hline & $51-100 \%$ & 6.7 & 6.7 & 3.3 & 0.0 & 13.3 \\
\hline & $0 \%$ & 0.0 & 0.0 & 3.3 & 0.0 & 0.0 \\
\hline & $1-10 \%$ & 70.0 & 70.0 & 63.3 & 76.7 & 76.7 \\
\hline & $11-20 \%$ & 23.3 & 20.0 & 30.0 & 13.3 & 6.7 \\
\hline Italy $(\mathrm{N}=30)$ & $21-30 \%$ & 3.3 & 6.7 & 0.0 & 3.3 & 13.3 \\
\hline & $31-40 \%$ & 0.0 & 0.0 & 0.0 & 0.0 & 0.0 \\
\hline & $41-50 \%$ & 3.3 & 3.3 & 3.3 & 6.7 & 0.0 \\
\hline & $51-100 \%$ & 0.0 & 0.0 & 0.0 & 0.0 & 3.3 \\
\hline & $0 \%$ & 16.7 & 13.3 & 6.7 & 3.3 & 0.0 \\
\hline & $1-10 \%$ & 60.0 & 66.7 & 70.0 & 70.0 & 33.3 \\
\hline & $11-20 \%$ & 3.3 & 6.7 & 10.0 & 10.0 & 33.3 \\
\hline $\begin{array}{l}\text { HIV physiclans in } \\
\text { the UK }(\mathrm{N}=30)\end{array}$ & $21-30 \%$ & 13.3 & 10.0 & 13.3 & 10.0 & 13.3 \\
\hline & $31-40 \%$ & 6.7 & 3.3 & 0.0 & 3.3 & 3.3 \\
\hline & $41-50 \%$ & 0.0 & 0.0 & 0.0 & 0.0 & 13.3 \\
\hline & $51-100 \%$ & 0.0 & 0.0 & 0.0 & 3.3 & 3.3 \\
\hline
\end{tabular}

time as my HIV treatment' had almost three-fold higher prevalence than those without this constraint, of missing ART because they 'were taking another medication' $(17.2 \%$ vs $6.0 \%$ respectively; $\mathrm{p}<0.001)$. Similarly, those reporting 'I must take food at the same time as my HIV treatment', had three-fold higher prevalence than those not on ART with food requirements, to miss ART dose because they had 'a problem taking pills at specified times (with meals, on empty stomach etc.)' $(22.5 \%$ vs $7.5 \%$ respectively; $\mathrm{p}<0.001$ ). PLHIV on regimens containing integrase strand transfer inhibitors were less likely to report that 'I must take food at the same time as my HIV treatment', compared to those on regimens without integrase strand transfer inhibitors (35.0\% [119/340] vs 53.2\% [126/237], respectively; $\mathrm{p}<0.001$ ). Conversely, reported food requirements with ART were significantly higher among those on regimens containing versus not containing protease inhibitors $(50.8 \%$ [62/122] vs $40.2 \%$ [183/455]; $\mathrm{p}=0.035$ ), or non-nucleoside reverse transcriptase inhibitors $(55.9 \%$ [114/204] vs $35.1 \%$ [131/373], respectively; $p<0.001)$. The percentage reporting 
Figure 3. Prevalence of specific disease conditions reported among people living with HIV on antiretroviral therapy, overall and by country, 2019

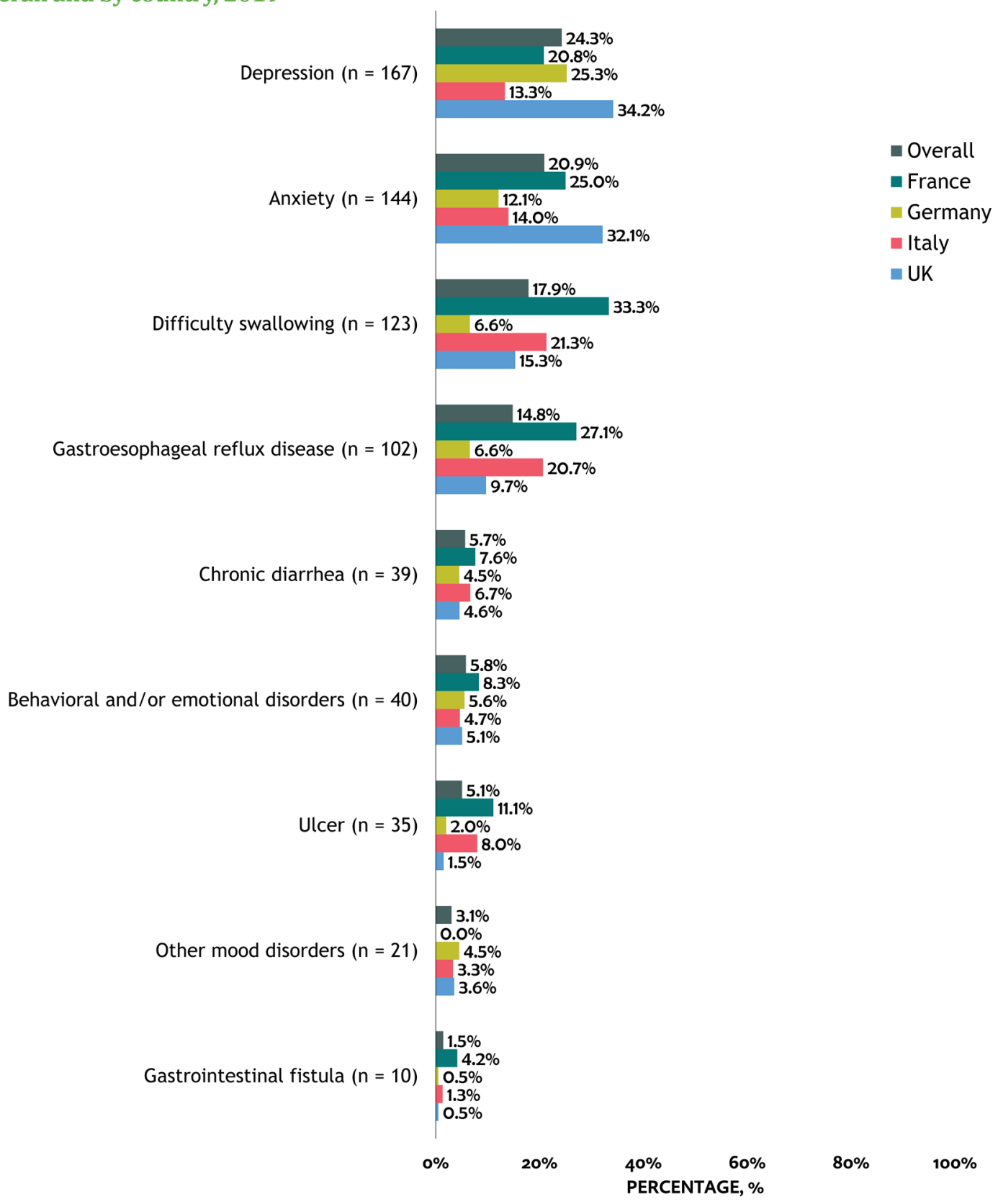

Note: Numbers in parenthesis beside each condition represent the total numerator within pooled analyses. Conditions with overall prevalence $<1 \%$ not shown in figure.

'I cannot take another drug at the same time as my HIV treatment' was significantly higher among those on regimens containing versus not containing protease inhibitors $(24.6 \%$ [30/122] vs $13.8 \%$ [63/455], respectively; $\mathrm{p}=0.004$ ), or nonnucleoside reverse transcriptase inhibitors $(20.6 \%$ [42/204] vs $13.7 \%$ [51/373], respectively; $\mathrm{p}=0.031$ ) (Table 3).

\section{Daily oral ART and challenges with adherence}

Of PLHIV on ART, 23.8\% [164/688] reported suboptimal adherence to daily oral ART (Figure 1); the corresponding estimate reported by HCPs was 33.6\%. Furthermore, $40.1 \%$ [276/688] of PLHIV reported being worried about missing their daily oral ART. PLHIV-reported average time (minutes) spent on daily oral ART administration was as follows: overall, 12.3 (SD=32.3); with malabsorption, 25.7 ( $\mathrm{SD}=52.4$ ); with interfering gastrointestinal conditions interfering, 24.9 ( $S D=47.7)$; with difficulty swallowing, 33.1 ( $S D=55.7)$; with neurocognitive conditions, $12.6(\mathrm{SD}=29.0)$; and with none of these four conditions, $6.3(\mathrm{SD}=16.6)$ minutes. The percentage of PLHIV needing help from someone to take their daily oral 
Table 3. Characteristics of people living with HIV on antiretroviral therapy in four European countries as well as the percentage reporting various medical conditions interfering with daily oral ART dosing and other treatment challenges, 2019

\begin{tabular}{|c|c|c|c|c|c|c|c|c|c|c|c|c|}
\hline \multirow[t]{2}{*}{ Characteristics } & \multicolumn{2}{|c|}{$\begin{array}{l}\text { Need monitoring when } \\
\text { taking other medications } \\
\text { with ART }\end{array}$} & \multicolumn{2}{|c|}{$\begin{array}{l}\text { Ever changed ART } \\
\text { because of DDI }\end{array}$} & \multicolumn{2}{|c|}{$\begin{array}{c}\text { Unable to take antacids } \\
\text { with ART }\end{array}$} & \multicolumn{2}{|c|}{$\begin{array}{l}\text { ART has food } \\
\text { requirements }\end{array}$} & \multicolumn{2}{|c|}{$\begin{array}{c}\text { Cannot take } \\
\text { another drug at } \\
\text { the same time with } \\
\text { ART }\end{array}$} & \multicolumn{2}{|c|}{$\begin{array}{l}\text { Ever hid or } \\
\text { disguised ART }\end{array}$} \\
\hline & $\%$ & $\mathbf{p}$ & $\%$ & $\mathbf{p}$ & $\%$ & $\mathbf{p}$ & $\%$ & $\mathbf{p}$ & $\%$ & $\mathbf{p}$ & $\%$ & $\mathbf{p}$ \\
\hline Total & 19.1 & & 15.4 & & 23.9 & & 42.5 & & 16.1 & & 43.3 & \\
\hline \multicolumn{13}{|l|}{ Gender } \\
\hline Women & 27.1 & \multirow{3}{*}{$\begin{array}{c}\chi^{2}(2)=11.51 \\
p=0.003\end{array}$} & 24.7 & \multirow{3}{*}{$\begin{array}{c}\chi^{2}(2)=16.12 \\
p<0.001\end{array}$} & 29.4 & \multirow{3}{*}{$\begin{array}{l}\chi^{2}(2)=4.52 \\
p=0.105\end{array}$} & 54.1 & \multirow{3}{*}{$\begin{array}{c}\chi^{2}(2)=16.61 \\
p<0.001\end{array}$} & 21.8 & \multirow{3}{*}{$\begin{array}{c}\chi^{2}(2)=5.97 \\
p=0.051\end{array}$} & 39.7 & \multirow{3}{*}{$\begin{array}{c}\chi^{2}(2)=1.81 \\
p=0.405\end{array}$} \\
\hline Men & 15.6 & & 11.6 & & 21.7 & & 37.3 & & 13.8 & & 45.1 & \\
\hline Other & बा & & Т & & T & & Т & & \} $&{ } &{\text { 【 }} &{ } \\
{\hline \multicolumn{13}{|c|}{\text { Sexual orientation }}\text { Sexual orientation }} \\
{\hline \text { Heterosexual }} &{24.9} &{\multirow{3}{*}{\begin{array}{c}\chi^{2}(2)=6.52 \\
p=0.038\end{array}}\begin{array} { c } { \chi ^ { 2 } ( 2 ) = 6 . 5 2 } \\
{ p = 0 . 0 3 8 } \end{array}} &{21.3} &{\multirow{3}{*}{\begin{array}{c}\chi^{2}(2)=9.81 \\
p=0.007\end{array}}\begin{array} { c } { \chi ^ { 2 } ( 2 ) = 9 . 8 1 } \\
{ p = 0 . 0 0 7 } \end{array}} &{26.6} &{\multirow{3}{*}{\begin{array}{c}\chi^{2}(2)=1.01 \\
p=0.602\end{array}}\begin{array} { c } { \chi ^ { 2 } ( 2 ) = 1 . 0 1 } \\
{ p = 0 . 6 0 2 } \end{array}} &{53.3} &{\multirow{3}{*}{\begin{array}{c}\chi^{2}(2)=12.22 \\
p=0.002\end{array}}\begin{array} { c } { \chi ^ { 2 } ( 2 ) = 1 2 . 2 2 } \\
{ p = 0 . 0 0 2 } \end{array}} &{17.2} &{\chi^{2}(2)=5.87} &{39.5} &{\multirow{3}{*}{\begin{array}{c}\chi^{2}(2)=3.30 \\
p=0.192\end{array}}\begin{array} { c } { \chi ^ { 2 } ( 2 ) = 3 . 3 0 } \\
{ p = 0 . 1 9 2 } \end{array}} \\
{\hline \text { Homosexual }} &{16} &{ } &{12.0} &{ } &{22.9} &{ } &{37.3} &{ } &{14.4} &{\mathrm{p}=0.053} &{46.0} &{ } \\
{\hline \text { Other }} &{24.2} &{ } &{24.2} &{ } &{21.2} &{ } &{45.5} &{ } &{30.3} &{ } &{36.8} &{ } \\
{\hline \multicolumn{13}{|l|}{\text { Age (years) }}\text { Age (years) }} \\
{\hline<50} &{20.4} &{\multirow{2}{*}{\begin{array}{c}\chi^{2}(1)=1.75 \\
p=0.186\end{array}}\begin{array} { c } { \chi ^ { 2 } ( 1 ) = 1 . 7 5 } \\
{ p = 0 . 1 8 6 } \end{array}} &{16.5} &{\multirow{2}{*}{\begin{array}{c}\chi^{2}(1)=1.37 \\
p=0.241\end{array}}\begin{array} { c } { \chi ^ { 2 } ( 1 ) = 1 . 3 7 } \\
{ p = 0 . 2 4 1 } \end{array}} &{26.8} &{\multirow{2}{*}{\begin{array}{c}\chi^{2}(1)=6.36 \\
p=0.012\end{array}}\begin{array} { c } { \chi ^ { 2 } ( 1 ) = 6 . 3 6 } \\
{ p = 0 . 0 1 2 } \end{array}} &{44.3} &{\multirow{2}{*}{\begin{array}{c}\chi^{2}(1)=1.94 \\
p=0.164\end{array}}\begin{array} { c } { \chi ^ { 2 } ( 1 ) = 1 . 9 4 } \\
{ p = 0 . 1 6 4 } \end{array}} &{19.0} &{\multirow{2}{*}{\begin{array}{c}\chi^{2}(1)=8.64 \\
p=0.003\end{array}}\begin{array} { c } { \chi ^ { 2 } ( 1 ) = 8 . 6 4 } \\
{ p = 0 . 0 0 3 } \end{array}} &{50.2} &{\multirow{2}{*}{\begin{array}{c}\chi^{2}(1)=31.58 \\
p<0.001\end{array}}\begin{array} { c } { \chi ^ { 2 } ( 1 ) = 3 1 . 5 8 } \\
{ p < 0 . 0 0 1 } \end{array}} \\
{\hline \geq 50} &{15.7} &{ } &{12.7} &{ } &{16.9} &{ } &{38.0} &{ } &{9.0} &{ } &{27.0} &{ } \\
{\hline \multicolumn{13}{|l|}{\text { Education level }}\text { Education level }} \\
{\hline \text { Postgraduate }} &{23.3} &{\multirow{4}{*}{\begin{array}{c}\chi^{2}(3)=5.21 \\
p=0.157\end{array}}\begin{array} { c } { \chi ^ { 2 } ( 3 ) = 5 . 2 1 } \\
{ p = 0 . 1 5 7 } \end{array}} &{13.3} &{\chi^{2}(3)=1.87} &{31.7} &{\chi^{2}(3)=7.68} &{43.3} &{\chi^{2}(3)=1.19} &{20.0} &{\chi^{2}(3)=6.29} &{64.2} &{\chi^{2}(3)=30.15} \\
{\hline \text { College }} &{19.6} &{ } &{16.6} &{\mathrm{p}=0.600} &{23.9} &{\mathrm{p}=0.053} &{43.9} &{\mathrm{p}=0.756} &{14.4} &{p=0.098} &{40.1} &{\mathrm{p}<0.001} \\
{\hline \text { Secondary }} &{12.3} &{ } &{12.3} &{ } &{17.3} &{ } &{43.2} &{ } &{21.0} &{ } &{34.3} &{ } \\
{\hline \text { Other }} &{11.4} &{ } &{20.0} &{ } &{14.3} &{ } &{34.3} &{ } &{5.7} &{ } &{34.1} &{ } \\
{\hline \text { Employment }} &{ } &{ } &{ } &{ } &{ } &{ } &{ } &{ } &{ } &{ } &{ } &{ } \\
{\hline \text { Employed }} &{18.3} &{\chi^{2}(1)=0.23} &{14.9} &{\chi^{2}(1)=0.34} &{25.5} &{\chi^{2}(1)=1.31} &{43.0} &{\chi^{2}(1)<0.01} &{15.6} &{\chi^{2}(1)=0.11} &{48.6} &{\chi^{2}(1)=14.25} \\
{\hline \text { Non-employed }} &{20} &{p=0.629} &{16.8} &{\mathrm{p}=0.558} &{21.1} &{\mathrm{p}=0.253} &{43.2} &{\mathrm{p}=0.951} &{16.8} &{p=0.737} &{33.0} &{\mathrm{p}<0.001} \\
{\hline \text { Country }} &{ } &{ } &{ } &{ } &{ } &{ } &{ } &{ } &{ } &{ } &{ } &{ } \\
{\hline \text { France }} &{30.4} &{\chi^{2}(3)=23.06} &{28.9} &{\chi^{2}(3)=24.56} &{36.3} &{\chi^{2}(3)=29.52} &{56.3} &{\chi^{2}(3)=14.40} &{18.5} &{\chi^{2}(3)=5.13} &{46.5} &{\chi^{2}(3)=23.09} \\
{\hline \text { Germany }} &{8.2} &{\mathrm{p}<0.001} &{10.9} &{\mathrm{p}<0.001} &{9.5} &{\mathrm{p}<0.001} &{37.4} &{\mathrm{p}=0.002} &{10.2} &{\mathrm{p}=0.163} &{29.8} &{\mathrm{p}<0.001} \\
{\hline \text { Italy }} &{21.3} &{ } &{11.0} &{ } &{28.3} &{ } &{36.2} &{ } &{18.1} &{ } &{54.0} &{ } \\
{\hline \text { UK }} &{17.9} &{ } &{11.9} &{ } &{23.2} &{ } &{40.5} &{ } &{17.9} &{ } &{46.4} &{ } \\
$\hline
\end{tabular}


Table 3. Continued

\begin{tabular}{|c|c|c|c|c|c|c|c|c|c|c|c|c|}
\hline \multirow[t]{2}{*}{ Characteristics } & \multicolumn{2}{|c|}{$\begin{array}{c}\text { Need monitoring when } \\
\text { taking other medications } \\
\text { with ART }\end{array}$} & \multicolumn{2}{|c|}{$\begin{array}{l}\text { Ever changed ART } \\
\text { because of DDI }\end{array}$} & \multicolumn{2}{|c|}{$\begin{array}{c}\text { Unable to take antacids } \\
\text { with ART }\end{array}$} & \multicolumn{2}{|c|}{$\begin{array}{l}\text { ART has food } \\
\text { requirements }\end{array}$} & \multicolumn{2}{|c|}{$\begin{array}{c}\text { Cannot take } \\
\text { another drug at } \\
\text { the same time with } \\
\text { ART }\end{array}$} & \multicolumn{2}{|c|}{$\begin{array}{l}\text { Ever hid or } \\
\text { disguised ART }\end{array}$} \\
\hline & $\%$ & $\mathbf{p}$ & $\%$ & $\mathbf{p}$ & $\%$ & $\mathbf{p}$ & $\%$ & $\mathbf{p}$ & $\%$ & $\mathbf{p}$ & $\%$ & $\mathbf{p}$ \\
\hline \multicolumn{13}{|l|}{ ART formulation } \\
\hline Single table & 18.2 & \multirow{2}{*}{$\begin{array}{l}\chi^{2}(1)=0.36 \\
p=0.549\end{array}$} & 13.5 & \multirow{2}{*}{$\begin{array}{c}\chi^{2}(1)=2.07 \\
p=0.150\end{array}$} & 24.8 & \multirow{2}{*}{$\begin{array}{c}\chi^{2}(1)=0.28 \\
p=0.595\end{array}$} & 47.0 & \multirow{2}{*}{$\begin{array}{c}\chi^{2}(1)=6.07 \\
p=0.014\end{array}$} & 16.3 & \multirow{2}{*}{$\begin{array}{c}\chi^{2}(1)=0.02 \\
p=0.894\end{array}$} & 45.7 & \multirow{2}{*}{$\begin{array}{c}\chi^{2}(1)=1.93 \\
p=0.165\end{array}$} \\
\hline Multi-tablet & 20.2 & & 17.8 & & 22.9 & & 36.8 & & 15.9 & & 40.4 & \\
\hline Non-suppressed & 31.2 & \multirow{2}{*}{$\begin{array}{l}\chi^{2}(1)=6.93 \\
p=0.008\end{array}$} & 34.4 & \multirow{2}{*}{$\begin{array}{c}\chi^{2}(1)=19.82 \\
p<0.001\end{array}$} & 42.2 & \multirow{2}{*}{$\begin{array}{c}\chi^{2}(1)=13.20 \\
p<0.001\end{array}$} & 53.1 & $\chi^{2}(1)=3.35$ & 34.4 & \multirow{2}{*}{$\begin{array}{c}\chi^{2}(1)=17.75 \\
p<0.001\end{array}$} & 54.8 & \multirow{2}{*}{$\begin{array}{c}\chi^{2}(1)=4.38 \\
p=0.036\end{array}$} \\
\hline Suppressed & 17.5 & & 13.1 & & 21.6 & & 41.1 & $p=0.067$ & 13.8 & & 42.0 & \\
\hline \multicolumn{13}{|l|}{ ART side effects ${ }^{a}$} \\
\hline None & 14.4 & \multirow{2}{*}{$\begin{array}{c}\chi^{2}(2)=13.50 \\
p=0.001\end{array}$} & 10.7 & \multirow{2}{*}{$\begin{array}{c}\chi^{2}(2)=23.56 \\
p<0.001\end{array}$} & 18.5 & \multirow{2}{*}{$\begin{array}{c}\chi^{2}(2)=8.77 \\
p=0.012\end{array}$} & 34.7 & \multirow{2}{*}{$\begin{array}{c}\chi^{2}(2)=15.55 \\
p<0.001\end{array}$} & 11.4 & \multirow{2}{*}{$\begin{array}{c}\chi^{2}(2)=13.10 \\
p=0.001\end{array}$} & 38.7 & \multirow{2}{*}{$\begin{array}{c}\chi^{2}(2)=12.00 \\
p=0.002\end{array}$} \\
\hline $\begin{array}{l}\text { Non-gastrointestinal } \\
\text { only }\end{array}$ & 13.8 & & 6.2 & & 26.2 & & 41.2 & & 12.5 & & 37.5 & \\
\hline \multicolumn{13}{|l|}{ HIV diagnosis year } \\
\hline 2017-19 & 29.4 & \multirow{3}{*}{$\begin{array}{c}\chi^{2}(2)=4.37 \\
p=0.113\end{array}$} & 7.8 & \multirow{3}{*}{$\begin{array}{c}\chi^{2}(2)=3.48 \\
p=0.175\end{array}$} & 33.3 & \multirow{3}{*}{$\begin{array}{c}\chi^{2}(2)=8.78 \\
p=0.012\end{array}$} & 56.9 & \multirow{3}{*}{$\begin{array}{c}\chi^{2}(2)=7.37 \\
p=0.025\end{array}$} & 19.6 & $\chi^{2}(2)=7.54$ & 37.5 & $\chi^{2}(2)=27.01$ \\
\hline $2010-16$ & 19.2 & & 17.7 & & 27.5 & & 44.5 & & 20.0 & $\mathrm{p}=0.023$ & 54.9 & $\mathrm{p}<0.001$ \\
\hline Pre-2010 & 16.9 & & 14.6 & & 18.4 & & 37.5 & & 11.5 & & 34.4 & \\
\hline $\begin{array}{l}\text { NNRTI-containing } \\
\text { ART }^{\text {b }}\end{array}$ & & & & & & & & & & & & \\
\hline No & 16.6 & $\chi^{2}(1)=4.08$ & 14.2 & $\chi^{2}(1)=1.19$ & 20.4 & $\chi^{2}(1)=7.27$ & 35.1 & $\chi^{2}(1)=23.27$ & 13.7 & $\chi^{2}(1)=4.66$ & 40.9 & $\chi^{2}(1)=3.12$ \\
\hline Yes & 23.5 & $\mathrm{p}=0.043$ & 17.6 & $\mathrm{p}=0.274$ & 30.4 & $\mathrm{p}=0.007$ & 55.9 & $\mathrm{p}<0.001$ & 20.6 & $\mathrm{p}=0.031$ & 47.9 & $\mathrm{p}=0.078$ \\
\hline $\begin{array}{l}\text { Entry inhibitor- } \\
\text { containing ART }\end{array}$ & & & & & & & & & & & & \\
\hline No & 18.5 & $\chi^{2}(1)=3.41$ & 14.0 & $\chi^{2}(1)=24.87$ & 23.7 & $\chi^{2}(1)=0.42$ & 42.2 & $\chi^{2}(1)=0.48$ & 16.0 & $\chi^{2}(1)=0.23$ & 43.4 & $\chi^{2}(1)=0.08$ \\
\hline Yes & 35 & $\mathrm{p}=0.065$ & 55.0 & $\mathrm{p}<0.001$ & 30.0 & $\mathrm{p}=0.516$ & 50.0 & $\mathrm{p}=0.488$ & 20.0 & $\mathrm{p}=0.631$ & 40.7 & $\mathrm{p}=0.783$ \\
\hline
\end{tabular}


Table 3. Continued

\begin{tabular}{|c|c|c|c|c|c|c|c|c|c|c|c|c|}
\hline \multicolumn{13}{|c|}{$\begin{array}{l}\text { INSTI-containing } \\
\text { ART }^{d}\end{array}$} \\
\hline No & 19.8 & \multirow{2}{*}{$\begin{array}{c}\chi^{2}(1)=0.15 \\
p=0.695\end{array}$} & 14.3 & \multirow{2}{*}{$\begin{array}{c}\chi^{2}(1)=0.36 \\
p=0.549\end{array}$} & 23.6 & \multirow{2}{*}{$\begin{array}{c}\chi^{2}(1)=0.02 \\
p=0.892\end{array}$} & 53.2 & \multirow{2}{*}{$\begin{array}{c}\chi^{2}(1)=18.86 \\
p<0.001\end{array}$} & 16.0 & \multirow{2}{*}{$\begin{array}{c}\chi^{2}(1)<0.01 \\
p=0.963\end{array}$} & 45.0 & \multirow{2}{*}{$\begin{array}{c}\chi^{2}(1)=0.62 \\
p=0.433\end{array}$} \\
\hline Yes & 18.5 & & 16.2 & & 24.1 & & 35.0 & & 16.2 & & 42.0 & \\
\hline \multicolumn{13}{|c|}{$\begin{array}{l}\text { Protease inhibitor- } \\
\text { containing ART }\end{array}$} \\
\hline No & 17.4 & \multirow{2}{*}{$\begin{array}{c}\chi^{2}(1)=4.04 \\
p=0.044\end{array}$} & 14.3 & \multirow{2}{*}{$\begin{array}{c}\chi^{2}(1)=2.14 \\
p=0.144\end{array}$} & 23.7 & \multirow{2}{*}{$\begin{array}{c}\chi^{2}(1)=0.04 \\
p=0.844\end{array}$} & 40.2 & \multirow{2}{*}{$\begin{array}{c}\chi^{2}(1)=4.42 \\
p=0.035\end{array}$} & 13.8 & \multirow{2}{*}{$\begin{array}{c}\chi^{2}(1)=8.21 \\
p=0.004\end{array}$} & 43.3 & \multirow{2}{*}{$\begin{array}{c}\chi^{2}(1)<0.01 \\
p=0.996\end{array}$} \\
\hline Yes & 25.4 & & 19.7 & & 24.6 & & 50.8 & & 24.6 & & 43.3 & \\
\hline \multicolumn{13}{|c|}{ Past ART resistance ${ }^{f}$} \\
\hline No & 18.1 & \multirow{2}{*}{$\begin{array}{c}\chi^{2}(1)=2.63 \\
p=0.105\end{array}$} & 13.1 & \multirow{2}{*}{$\begin{array}{c}\chi^{2}(1)=16.57 \\
p<0.001\end{array}$} & 23.0 & \multirow{2}{*}{$\begin{array}{c}\chi^{2}(1)=1.78 \\
p=0.183\end{array}$} & 41.7 & \multirow{2}{*}{$\begin{array}{c}\chi^{2}(1)=1.03 \\
p=0.310\end{array}$} & 14.7 & \multirow{2}{*}{$\begin{array}{c}\chi^{2}(1)=6.07 \\
p=0.014\end{array}$} & 42.6 & \multirow{2}{*}{$\begin{array}{c}\chi^{2}(1)=0.96 \\
p=0.328\end{array}$} \\
\hline Yes & 26 & & 31.5 & & 30.1 & & 47.9 & & 26.0 & & 48.2 & \\
\hline
\end{tabular}

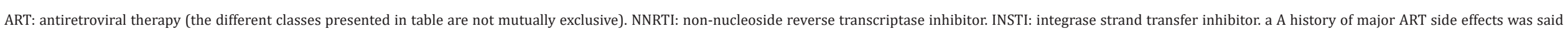

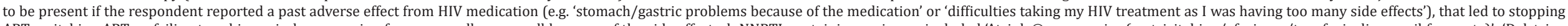

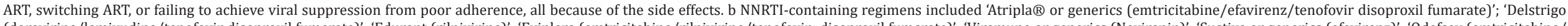

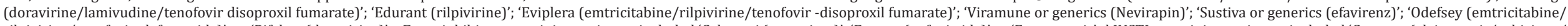

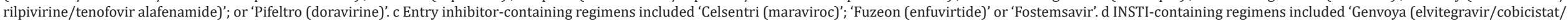

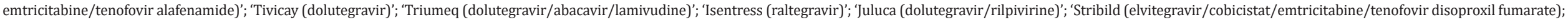

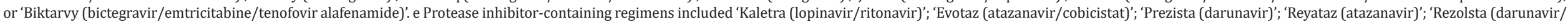

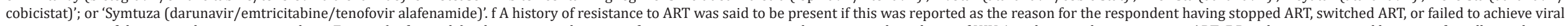

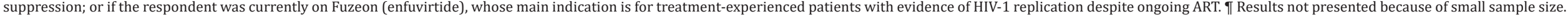


ART was as follows: with malabsorption, $17.0 \%(9 / 53)$; with interfering gastrointestinal condition, $14.8 \%(19 / 128)$; and with neurocognitive conditions, $16.1 \%(38 / 236)$. The percentage spending more time or effort to take their daily oral ART because of their condition was: with malabsorption, $30.2 \%$ (16/53); with interfering gastrointestinal condition, $35.2 \%(45 / 128)$; and with neurocognitive conditions, $33.9 \%$ (80/236). According to HCPs, patients 'not taking HIV medication as prescribed (non-adherent) for any nonmedical reasons (e.g. stigma, lifestyle, recreational drug use, pill fatigue, cost, life event)', was the leading reason 'for not being suppressed' (48.3\%) (Figure 4). Other perceived reasons 'for not being suppressed', from an HCP perspective, included patient 'not taking HIV medication as prescribed (non-adherent) because of tolerability issues' (38.8\%), 'patient non-adherence due to fear of long-term toxicities' (32.8\%), or because 'patient's virus became resistant to HIV treatment' (19.0\%). The top reasons reported by PLHIV who were virally non-suppressed $(n=68)$ included 'difficulties taking my HIV treatment as I was having too many side effects' (30.9\%; 21/68), 'the virus became resistant' (29.4\%; 20/68), 'higher viral load before starting HIV therapy' (27.9\%; 19/68), and 'difficulties taking my HIV pill (s) every day for any reason except another medical condition' $26.5 \%$; 18/68).
Confidentiality concerns associated with daily oral ART Among PLHIV, openness in sharing HIV status varied, from $29.7 \%(204 / 688)$ who reported that 'no one knows about my HIV status'; to $20.9 \%(144 / 688)$ who indicated 'I've limited what I tell others about my HIV', to $7.6 \%(52 / 688)$ who reported being 'generally open in talking about my status'. The percentage of PLHIV who reported they had shared their HIV status within various relationships was as follows, as applicable: with a partner/spouse/significant-other (91.0\%; 424/466), with other sexual partners (75.6\%; 391/517), with parents/siblings/children (78.0\%; 478/613), with wider family members (55.0\%; 333/605), with close friends (85.4\%; 534/625), with current family doctor (89.8\%; $561 / 625)$, with other healthcare professionals such as nurses, counsellors, pharmacists, and psychiatrists $(84.0 \%$; $516 / 614)$, with current employer $(33.4 \% ; 167 / 500)$, and with co-workers at current workplace $(37.5 \% ; 190 / 507)$. To avoid sharing their HIV status, $43.3 \%$ (298/688) reported hiding their HIV medications at least once within the past 6 months; over half of those who hid their medication $153.7 \%$; $160 / 298$ ) admitted they would feel stressed/anxious if someone [they] did not want to see [their] HIV pills were to find them'. Overall, $16.1 \%(111 / 688)$ reported that 'keeping my pills at home or with me during the day, I have been worried someone would see them and know about my

Figure 4. Comparison of PLHIV-reported versus physician-reported barriers to achieving viral suppression among persons living with HIV in four European countries, 2019

Non-adherence due to fear of long-term toxicities (e.g. liver, bones, kidneys)

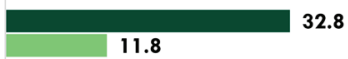

Recreational drug use (e.g. crystal meth, mephedrone, GBL, heroin, cocaine, cannabis) interaction and adherence issues

Comorbidities that interfere with dailv oral intake and bioavailability (e.g. difficulty swallowing)

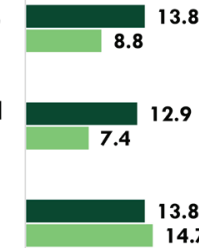

12.9

HCP-reported $(n=116)$

Difficulties taking food with HIV treatment 4.7 - PLHIV-reported $(n=68)$

Higher viral load before starting HIV therapy

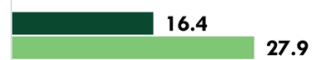

Non-adherence because of tolerability issues (frequency or severity of side effects)

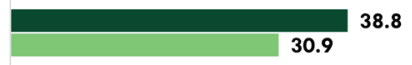

Drug-drug interactions of HIV treatment with other medications taken for other conditions/illnesses (excluding recreational drugs)

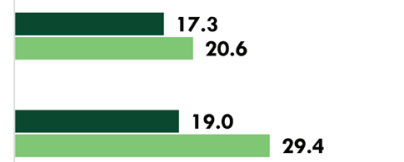

Non-adherence for any non-medical reasons (e.g. stigma, lifestyle, recreational drug use, pill fatigue, cost, life event)

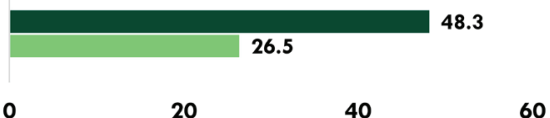

80

100 
Figure 5. Prevalence of emotional and psychosocial challenges to daily oral ART administration among people living with HIV on antiretroviral therapy in four European countries, stratified by experience of ART side effects, 2019

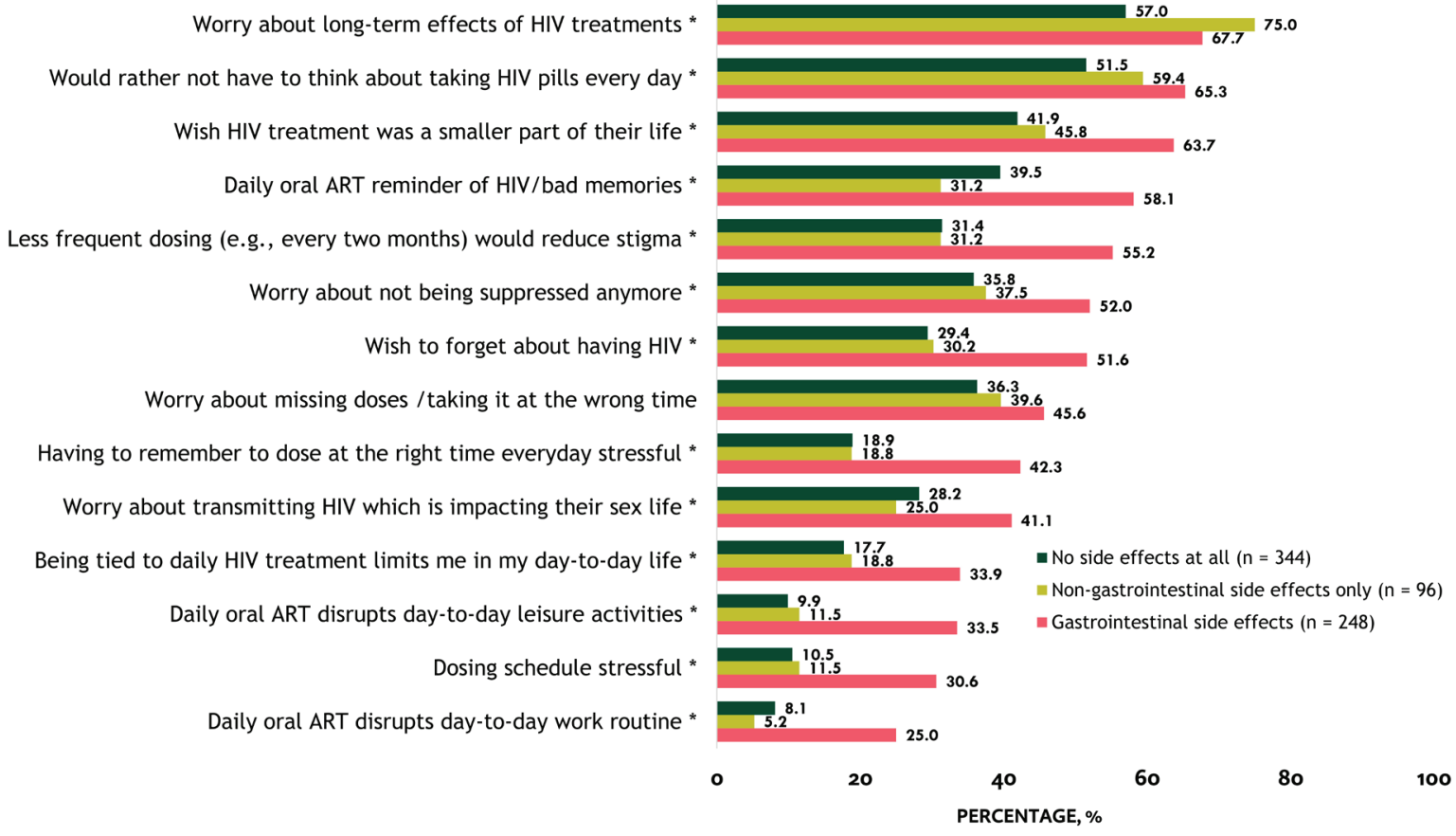

* Statistically significant differences within the three groups overall, based on chi-squared tests at $\mathrm{p}<0.05$.

HIV'. Specific situations where PLHIV were worried about unwanted disclosure of their HIV status included: while travelling at airports, by airport security or customs $(22.2 \%$; 153/688); while on holiday, by friends (15.1\%; 104/688); while at the workplace, by coworkers $(17.2 \% ; 118 / 688)$; or while at home, by family members $(10.2 \% ; 70 / 688)$. One reason reported by PLHIV for not wanting to share their HIV status was worry 'that I'll lose my source of income if other people find out that I have HIV' (16.7\%; 115/688). Overall, $16.6 \%(114 / 688)$ reported having missed ART doses because they 'were not in a situation where [they] felt comfortable taking [their] pills (privacy/confidentiality)'; 40.0\% (275/688) felt that 'taking my HIV treatment less often (for instance every 2 months instead of every day) would reduce the shame or stigma I feel for having HIV'.

Emotional wellbeing related to daily tablet requirements Overall, 45.1\% (310/688) of PLHIV felt that 'taking daily HIV treatment reminds me that I have HIV and/or of a mistake or bad memory from my past', and 50.3\% (346/688) wished 'my HIV treatment was a smaller part of my life'. Many felt that 'being tied to my daily HIV treatment limits me in my day-to-day life' $(23.7 \%$; 163/688). Furthermore, 27.3\% $(188 / 688)$ felt that 'having to remember to take my HIV treatment at the right time every day causes me stress or anxiety'; 57.6\% (396/688) 'would rather not have to think about taking the pills every day'; $41.9 \%(288 / 688)$ reported 'worry about missing doses and not being suppressed anymore'; while 32.4\% (223/688) indicated 'I worry about missing doses and transmitting the disease which is impacting my sex life'. These concerns were particularly pronounced among those experiencing gastrointestinal side effects from their ART (Figure 5). Differences existed on some measures between PLHIV and HCP estimates. For example, 63.4\% (436/688) of PLHIV reported being worried about long-term effects of HIV treatment, almost twice the estimate reported by HCPs in relation to HIV patients (33.3\%) (Figure 1).

\section{DISCUSSION}

We found that a substantial proportion of PLHIV reported several problems related to daily oral dosing, including medical conditions that limit oral administration, emotional challenges (e.g. pill fatigue), privacy and confidentiality concerns, all of which collectively contribute to poor adherence. While HCPs were also aware of the confidentiality and emotional concerns faced by patients, HCPs' estimates of these issues were lower than those reported by PLHIV. This suggests that not all patients discuss HIV-related emotional challenges with their $\mathrm{HCPs}^{27}$. The gap between perceived and actual emotional wellbeing among PLHIV underscores the urgent need to address the stigma still surrounding HIV to improve quality of life ${ }^{32-34}$. Such stigma may explain findings from previous studies showing that on dimensions of mental 
health and psychological factors, PLHIV show a detriment versus the general population ${ }^{35}$. Consistent with our findings, previous research has documented patients' worries that their HIV status may be disclosed inadvertently if they store their antiretroviral medications at home and take them at regular intervals; therefore, patients have reported skipping or delaying doses to avoid stigma ${ }^{12}$.

The percentage of PLHIV with suboptimal adherence in our study (23.8\%) is very close to the $24.1 \%$ overall estimate reported in a recent multi-country study ${ }^{12}$. Another study by Okoli et al. ${ }^{36}$ found that $66.6 \%$ of PLHIV worried about the long-term effects of HIV medicines, similar to $63.4 \%$ in our study. That same study reported that $18.6 \%$ (288/1550) of those who ever switched ART (or 13.6\% [288/2112] of all study participants) reported switching because of drug-drug interactions; in our study, $15.4 \%$ of all participants reported ever changing ART because of drug-drug interactions. Regarding confidentiality concerns, our finding that only $7.6 \%$ of participants reported being fully open in discussing their HIV status is similar to the estimate of $6.8 \%$ reported in the 2019 Positive Perspectives Survey from 25 countries regarding the percentage of PLHIV who 'always' shared their HIV status ${ }^{37}$. The population subgroups with the highest prevalence of swallowing difficulty in our study included women, younger adults, those on multi-tablet regimens, and those experiencing gastrointestinal side effects. Some of these subgroups have been identified in previous research as being at highest risk for suboptimal adherence ${ }^{12}$.

Current approaches to improving the ease of drug administration for patients with dysphagia or pill aversion include reducing the number of pills and altering the drug form ${ }^{38}$. The use of small pills has been effective for suppressing viral load in some patients with dysphagia ${ }^{39}$. Crushing pills or opening capsules may be acceptable in some cases; however, many antiretroviral agents are not recommended for crushing (if a pill) or opening (if a capsule) because the change in the formulation can reduce its bioavailability and targeted therapeutic exposure ${ }^{40}$. New non-oral long-acting directly observed ARTs could potentially address some of the unmet problems identified in this study. Indeed, previous studies show that many PLHIV believe that a long-acting ART would address some of the challenges they face with daily oral ART ${ }^{20,21}$.

\section{Strengths and limitations}

The strength of this study is that we have explored both PLHIV and HCP perspectives regarding challenges to daily oral dosing in four European countries. Self-reported HIV diagnosis was followed by a confirmed ascertainment of HIV status for all PLHIV. Sampling was done such that the distribution of participants on key demographic variables matched national statistics for all PLHIV. Nonetheless, limitations exist. First, the HCP and PLHIV data in each country may not be directly comparable as the institutions from which the HCPs were mostly sampled may not necessarily reflect places where sampled PLHIV routinely access care. Second, these are cross-sectional analyses and only associations can be drawn. Third, neither the HCP nor PLHIV data may be fully representative of the respective countries or region, because of the non-probabilistic sampling.

\section{CONCLUSIONS}

Many treatment-related problems were identified in relation to daily intake of oral medication among PLHIV. HCPs estimated that approximately $10-15 \%$ of their patients were affected by each medical condition identified as interfering with daily oral administration. HCPs identified 'nonadherence for any non-medical reason' as the primary cause of virologic failure. Of surveyed PLHIV, 43.3\% reported hiding their medication, while $45.1 \%$ saw their tablets as a daily reminder of HIV. New non-oral long-acting directly observed ARTs could help address these problems and improve longterm outcomes among PLHIV.

\section{REFERENCES}

1. Mills EJ, Nachega JB, Buchan I, et al. Adherence to antiretroviral therapy in sub-Saharan Africa and North America: a meta-analysis. JAMA. 2006;296(6):679-690. doi:10.1001/jama.296.6.679

2. Iacob SA, Iacob DG, Jugulete G. Improving the adherence to antiretroviral therapy, a difficult but essential task for a successful HIV treatment-clinical points of view and practical considerations. Front Pharmacol. 2017;8:831. doi:10.3389/fphar.2017.00831

3. Nachega JB, Marconi VC, van Zyl GU, et al. HIV treatment adherence, drug resistance, virologic failure: evolving concepts. Infect Disord Drug Targets. 2011;11(2):167-174. doi:10.2174/187152611795589663

4. Nachega JB, Hislop M, Dowdy DW, et al. Adherence to highly active antiretroviral therapy assessed by pharmacy claims predicts survival in HIV-infected South African adults. J Acquir Immune Defic Syndr. 2006;43(1):78-84. doi:10.1097/01.qai.0000225015.43266.46

5. Joint United Nations Programme on HIV/AIDS. 90-90-90: An ambitious treatment target to help end the AIDS epidemic. Geneva, Switzerland: UNAIDS; 2014. https://www.unaids. org/en/resources/909090. Accessed March 30, 2020.

6. Yu Y, Luo D, Chen X, et al. Medication adherence to antiretroviral therapy among newly treated people living with HIV. BMC Public Health. 2018;18(1):825. doi:10.1186/s12889-018-5731-z

7. Durvasula R. HIV/AIDS in older women: unique challenges, unmet needs. Behav Med. 2014;40(3):85-98. doi:10.1080/08964289.2014.893983

8. Lennon CA, Pellowski JA, White AC, et al. Service priorities and unmet service needs among people living with HIV/ AIDS: results from a nationwide interview of HIV/AIDS housing organizations. AIDS Care. 2013;25(9):1083-1091. doi:10.1080/09540121.2012.749337 
9. Sok P, Gardner S, Bekele T, et al. Unmet basic needs negatively affect health-related quality of life in people aging with HIV: results from the Positive Spaces, Healthy Places study. BMC Public Health. 2018;18(1):644. doi:10.1186/s12889-018-5391-z

10. Beach SR, Schulz R, Friedman EM, et al. Adverse Consequences of Unmet Needs for Care in High-Need/ High-Cost Older Adults. J Gerontol B Psychol Sci Soc Sci. 2020;75(2):459-470. doi:10.1093/geronb/gby021

11. European Centre for Disease Prevention and Control, World Health Organization. HIV/AIDS surveillance in Europe 2019: 2018 Data. https://www.ecdc.europa.eu/sites/default/files/ documents/2019-Annual_HIV_Report_Cover\%2BInner-web. pdf. Published 2019. Accessed November 30, 2019.

12. de los Rios P, Okoli C, Punekar Y, et al. Prevalence, determinants, and impact of suboptimal adherence to HIV medication in 25 countries. Prev Med. 2020;139:106182. doi:10.1016/j.ypmed.2020.106182

13. Loutfy M, Johnson M, Walmsley S, et al. The association between HIV disclosure status and perceived barriers to care faced by women living with HIV in Latin America, China, Central/Eastern Europe, and Western Europe/ Canada. AIDS Patient Care and STDs. 2016;30(9):435-444. doi:10.1089/apc.2016.0049

14. Rydström LL, Eriksson LE, Berlin A. 'The Medication always Reminds Me'. Living with Perinatal acquired HIV-Children and Parents' View Points. Madridge Journal of AIDS. 2019;3(1):62-68. doi:10.18689/mja-1000111

15. Marzolini C, Back D, Weber R, et al. Ageing with HIV: medication use and risk for potential drug-drug interactions. J Antimicrob Chemother. 2011;66(9):2107-2111. doi:10.1093/jac/dkr248

16. Holtzman C, Armon C, Tedaldi E, et al. Polypharmacy and risk of antiretroviral drug interactions among the aging HIVinfected population. Journal of General Internal Medicine. 2013;28(10):1302-1310. doi:10.1007/s11606-013-2449-6

17. Dorman RM, Sutton SH, Yee LM. Understanding HIV-Related Pill Aversion as a Distinct Barrier to Medication Adherence. Behav Med. 2019;45(4):294-303. doi:10.1080/08964289.2018.1534076

18. Margolis DA, Gonzalez-Garcia J, Stellbrink HJ, et al. Long-acting intramuscular cabotegravir and rilpivirine in adults with HIV-1 infection(LATTE-2): 96-week results of a randomised, open-label, phase 2b, non-inferiority trial. Lancet. 2017;390(10101):14991510. doi:10.1016/S0140-6736(17)31917-7

19. Margolis DA, Brinson CC, Smith GHR, et al. Cabotegravir plus rilpivirine, once a day, after induction with cabotegravir plus nucleoside reverse transcriptase inhibitors in antiretroviralnaive adults with HIV-1 infection(LATTE): a randomised, phase 2b, dose-ranging trial. Lancet Infect Dis. 2015;15(10):11451155. doi:10.1016/S1473-3099(15)00152-8

20. de los Rios P, Okoli C, Young B, et al. Treatment Aspirations and Attitudes Towards Innovative Medications Among People Living with HIV in 25 Countries. Popul Med. 2020;2(July):1-13. doi:10.18332/popmed/124781

21. Kerrigan D, Mantsios A, Gorgolas M, et al. Experiences with long acting injectable ART: a qualitative study among
PLHIV participating in a Phase II study of cabotegravir + rilpivirine(LATTE-2) in the United States and Spain. PloS one. 2018;13(1):e0190487. doi:10.1371/journal.pone.0190487

22. Horne R, Cooper V, Gellaitry G, et al. Patients' perceptions of highly active antiretroviral therapy in relation to treatment uptake and adherence: the utility of the necessity-concerns framework. J Acquir Immune Defic Syndr. 2007;45(3):334341. doi:10.1097/QAI.0b013e31806910e3

23. Kalichman S, Kalichman MO, Cherry C. Medication beliefs and structural barriers to treatment adherence among people living with HIV infection. Psychol Health. 2016;31(4):383395. doi:10.1080/08870446.2015.1111371

24. Gonzalez JS, Penedo FJ, Llabre MM, et al. Physical symptoms, beliefs about medications, negative mood, and long-term HIV medication adherence. Ann Behav Med. 2007;34(1):46-55. doi:10.1007/BF02879920

25. Valverde E, Beer L, Johnson C, et al. Prevention counseling practices of HIV care providers with patients new to HIV medical care: medical monitoring project provider survey, 2009. J Int Assoc Provid AIDS Care. 2014;13(2):127-134. doi:10.1177/2325957413516496

26. Shaw S, Modi R, Mugavero M, et al. HIV Standard of Care for ART Adherence and Retention in Care Among HIV Medical Care Providers Across Four CNICS Clinics in the US. AIDS Behav. 2019;23(4):947-956. doi:10.1007/s10461-018-2320-1

27. Ddumba-Nyanzi I, Kaawa-Mafigiri D, Johannessen H. Barriers to communication between HIV care providers(HCPs) and women living with HIV about child bearing: A qualitative study. Patient Educ Couns. 2016;99(5):754-759. doi:10.1016/j.pec.2015.11.023

28. Mallinson RK, Rajabiun S, Coleman S. The provider role in client engagement in HIV care. AIDS Patient Care STDS. 2007;21(Suppl 1):S77-S84. doi:10.1089/apc.2007.9984

29. U.S. Department of Health \& Human Services. National HIV/AIDS Strategy for The United States: Updated to 2020. https://files.hiv.gov/s3fs-public/nhas-update.pdf. Published 2015. Accessed Feb 29, 2020.

30. Brown AE, Hayes R, Noori T, et al. HIV in Europe and Central Asia: progress in 2018 towards meeting the UNAIDS 90-90-90 targets. Euro Surveill. 2018;23(48):1800622. doi:10.2807/1560-7917.ES.2018.23.48.1800622

31. Roche Laboratories Inc., Trimeris Inc. Fuzeon(enfurvitide) for injection. https://www.accessdata.fda.gov/drugsatfda_ docs/label/2003/021481lbl.pdf. Published 2003. Accessed Feb 29, 2020.

32. Rice WS, Turan B, Fletcher FE, et al. A Mixed Methods Study of Anticipated and Experienced Stigma in Health Care Settings Among Women Living with HIV in the United States. AIDS Patient Care STDS. 2019;33(4):184-195. doi:10.1089/apc.2018.0282

33. Chan BT, Tsai AC. HIV stigma trends in the general population during antiretroviral treatment expansion: analysis of 31 countries in sub-Saharan Africa, 20032013. J Acquir Immune Defic Syndr. 2016;72(5):558-564. doi:10.1097/QAI.0000000000001011 
34. Lazarus JV, Safreed-Harmon K, Barton SE, et al. Beyond viral suppression of HIV - the new quality of life frontier. BMC Med. 2016;14(1):94. doi:10.1186/s12916-016-0640-4

35. Miners A, Phillips A, Kreif N, et al. Health-related qualityof-life of people with HIV in the era of combination antiretroviral treatment: a cross-sectional comparison with the general population. Lancet HIV. 2014;1(1):e32-40. doi:10.1016/S2352-3018(14)70018-9

36. Okoli C, de Los Rios P, Eremin A, et al. Relationship Between Polypharmacy and Quality of Life Among People in 24 Countries Living With HIV. Prev Chronic Dis. 2020;17:E22. doi:10.5888/pcd17.190359

37. Okoli C, Van de Velde N, Richman B, et al. Undetectable Equals Untransmittable $(\mathrm{U}=\mathrm{U})$ : Awareness and associations with health outcomes among people living with HIV in 25 countries. Sex Transm Infect. 2020. doi:10.1136/sextrans-2020-054551

38. Engelhard E, Smith C, Vervoort S, et al. Patients' willingness to take separate component antiretroviral therapy regimens for HIV in the Netherlands. J Int AIDS Soc. 2014;17(4Suppl 3):19536. doi:10.7448/IAS.17.4.19536

39. Suzuki T, Hara N, Osa M, et al. Efficacy of switching to dolutegravir plus rilpivirine, the small-tablet regimen, in patients with dysphagia: two case reports. J Pharm Health Care Sci. 2017;3:23. doi:10.1186/s40780-017-0093-8

40. Best BM, Capparelli EV, Diep H, et al. Pharmacokinetics of lopinavir/ritonavir crushed versus whole tablets in children. J Acquir Immune Defic Syndr. 2011;58(4):385-391. doi:10.1097/QAI.0b013e318232b057

\section{ACKNOWLEDGEMENTS}

Data collection was supported by Ipsos Healthcare. Data analyses and medical writing services were supported by Zatum LLC.

\section{CONFLICTS OF INTEREST}

The authors have each completed and submitted an ICMJE form for disclosure of potential conflicts of interest. The authors declare that they have no competing interests, financial or otherwise, related to the current work. L. A. Clark was a full-time employee at Ipsos Insights, LLC, during the conduct of the study. C. Karki and J. Noone report personal fees from Ipsos Insights, LLC during the conduct of the study.
L. Roustand, V. Chounta and N. Van de Velde report other grants from GlaxoSmithKline, outside the submitted work. L. Finkielsztejn, V. Chounta and N. Van de Velde report personal fees from ViiV Healthcare, outside the submitted work. G. Nachbaur reports that she is an employee and holds shares of GlaxoSmithKline, during the conduct of the study.

\section{FUNDING}

The research was funded by ViiV Healthcare.

PROVENANCE AND PEER REVIEW

Not commissioned; externally peer reviewed. 